\title{
Cullin-3-dependent deregulation of ACTN1 represents a pathogenic mechanism in nemaline myopathy
}

\author{
Jordan Blondelle, ${ }^{1}$ Kavya Tallapaka, Jane T. Seto, ${ }^{2,3}$ Majid Chassemian, ${ }^{4}$ Madison Clark, ${ }^{1}$ \\ Jenni M. Laitila, ${ }^{5}$ Adam Bournazos, ${ }^{6,7}$ Jeffrey D. Singer, ${ }^{8}$ and Stephan Lange ${ }^{1,9}$ \\ 'Division of Cardiology, School of Medicine, UCSD, La Jolla, California, USA. ${ }^{2}$ Neuromuscular Research, Murdoch Children's \\ Research Institute, Royal Children's Hospital, Parkville, Victoria, Australia. ${ }^{3}$ Department of Paediatrics, University of \\ Melbourne, Parkville, Victoria, Australia. ${ }^{4}$ Department of Chemistry and Biochemistry. UCSD, La Jolla, California, USA. \\ ${ }^{5}$ Folkhälsan Research Center and Medicum, University of Helsinki, Helsinki, Finland. ${ }^{6}$ Kids Neuroscience Centre, Kids \\ Research, Children's Hospital at Westmead, Sydney, New South Wales, Australia. 'Discipline of Child and Adolescent \\ Health, Faculty of Medicine and Health, The University of Sydney, Sydney, New South Wales, Australia. ${ }^{8}$ Department of \\ Biology, Portland State University, Portland, Oregon, USA. ' Wallenberg Laboratory, Department of Molecular and Clinical \\ Medicine, Institute of Medicine, University of Gothenburg, Gothenburg, Sweden.
}

Nemaline myopathy is a congenital neuromuscular disorder characterized by muscle weakness, fiber atrophy, and presence of nemaline bodies within myofibers. However, understanding of the underlying pathomechanisms is lacking. Recently, mutations in KBTBD13, KLHL40, and KLHL41, three substrate adaptors for the E3 ubiquitin ligase Cullin-3, have been associated with early-onset nemaline myopathies. We hypothesized that deregulation of Cullin-3 and its muscle protein substrates may be responsible for disease development. Using Cullin-3-knockout mice, we identified accumulation of non-muscle $\alpha$-actinins (ACTN1 and ACTN4) in muscles of these mice, which we also observed in patients with mutations in KBTBD13. Our data reveal that proper regulation of Cullin-3 activity and ACTN1 levels is essential for normal muscle and neuromuscular junction development. While ACTN1 is naturally downregulated during myogenesis, its overexpression in C2C12 myoblasts triggered defects in fusion, myogenesis, and acetylcholine receptor clustering - features that we characterized in Cullin-3-deficient mice. Taken together, our data highlight the importance of Cullin-3-mediated degradation of ACTN1 for muscle development, and indicate what is to our knowledge new pathomechanism for the etiology of myopathies seen in Cullin-3-knockout mice and patients with nemaline myopathy.

Conflict of interest: The authors have declared that no conflict of interest exists.

Copyright: @ 2019 American Society for Clinical Investigation

Submitted: October 22, 2018

Accepted: April 11, 2019

Published: April 16, 2019

Reference information: /CI Insight. 2019;4(10):e125665. https://doi. org/10.1172/ji.insight.125665

\section{Introduction}

Skeletal muscle mass represents up to $40 \%$ of total body weight in humans. Its maintenance is regulated by the equilibrium between muscle growth or physiological hypertrophy and muscle atrophy, reflecting the balance between protein synthesis and degradation at the cellular level. This balance is affected in pathologies such as cancer, HIV, and diabetes, leading to muscle atrophy and reduced life span (1). Accumulation of undegraded proteins in aggresomes or inclusion bodies is often associated with development of skeletal muscle myopathies, such as nemaline or reducing-body myopathy (2). Indeed, there is mounting evidence that the deregulation of protein turnover is the primary pathogenic mechanism for some forms of skeletal myopathies. Hence, a deeper understanding of the mechanisms that govern muscle protein degradation may contribute to the development of treatments and prevention of these pathologies.

Muscle cells contain 2 major systems for protein degradation: the autophagy-lysosome system and the ubiquitin-proteasome system (UPS). The latter is responsible for the degradation of $80 \%$ of muscle proteins. The UPS requires the tagging of substrates by polyubiquitin chains via an enzymatic cascade (3). First, the E1-activating enzyme covalently attaches to ubiquitin through an ATP-driven step. Once activated, ubiquitin is transferred to E2-conjugating enzymes. The last step requires the concerted action of E2-enzymes and E3 ligases, which transfer ubiquitin from the E2-enzyme onto the substrate (3). Once polyubiquitylated, the substrate is recognized by the proteasome for degradation. 
Cullin-RING ligases (CRLs) represent the largest E3 ubiquitin ligase family in mammals $(4,5)$. Cullin $(1-3,4 a, 4 b, 5,7,9)$ proteins are nearly ubiquitously expressed (6) and constitute the backbone of this complex. In order to form the functioning CRL complex, each Cullin binds to one of 2 RING (really interesting new gene) domain proteins ( $\mathrm{Rbx} 1$ or $\mathrm{Rbx} 2)$ that associates with E2-conjugating enzymes. In addition, Cullins interact with specific substrate adaptor protein families, thereby achieving specificity for a range of cellular substrates (7). The activity of CRLs is regulated through posttranslational modification of the Cullin backbone by the small ubiquitin-like modifier Nedd8, also called neddylation (7). Cullin neddylation is removed by the COP9 signalosome complex, leading to the inactivation of the CRL (8).

For the last decades, Cullin proteins were mainly studied in the context of cancer development due to their roles in the regulation of cell proliferation through the degradation of cyclin proteins (9). However, data regarding their roles in other tissues or pathologies remain poorly characterized. Few muscle-specific substrate adaptors that interact specifically with Cullin-1 have been intensively investigated. These include atrogin-1, which was found to play key roles during muscle atrophy (10). We have recently shown that neddylation and CRL activity are necessary for normal myoblast differentiation, fusion, and maturation in vitro (11).

Cullin-3 is highly enriched in muscle tissues (6), localizes to myofilaments, and is involved in muscle protein breakdown in adult animals (12). Cullin-3 utilizes the large family of BTB domain-containing proteins as substrate adaptors. A prominent subset of the BTB protein family with roles in skeletal muscles is the BTB/Kelch adaptor protein family, characterized by their combination of BTB and Kelch repeat domains (13). Proteins of this family are involved in a plethora of molecular and cellular mechanisms, such as cell migration, morphology, and protein expression (13). Very recently, mutations in several BTB/Kelch genes (i.e., KLHL9, KLHL40, KLHL41, and KBTBD13) were associated with the development of distal and nemaline myopathies in patients (13-17). The early onset of these myopathies suggests important roles for Cullin-3 and its associated protein turnover for skeletal muscle development.

While many BTB/Kelch domain proteins may be Cullin-3 substrate adaptors, their substrates in skeletal muscles remain largely unknown. However, KLHL40 was recently shown to be necessary for postnatal muscle growth through the degradation of dimerization partner 1 (DP1) and the subsequent repression of E2F1-DP1 complex activity (18). Unexpectedly, KLHL40 also acts as a stabilizer of the thin filament proteins leiomodin-3 and nebulin (19). This last discovery suggests noncanonical functions for the Cullin-3 E3 ligase complex that could also be important for the stabilization of some substrate proteins rather than their degradation. These findings add a new degree of complexity to the protein turnover mechanism related to Cullin-3. Therefore, the discovery of new substrates for Cullin-3 during muscle development will be of great interest for a better understanding of myogenesis and pathogenesis of associated myopathies. While their functions are mainly unknown in muscles, expression patterns of other BTB/Kelch domain proteins suggest important roles during muscle development (13). These findings represent an emerging role for Cullin-3 in muscle homeostasis. Indeed, characterizing the specific function(s) of Cullin-3 and its related protein turnover during muscle development would increase our understanding of muscle biology as well as pathological mechanisms for the etiology of muscle diseases such as nemaline myopathy.

During preparation of the present manuscript, an article investigating the role of Cullin-3 in striated muscles was published (20). The authors demonstrated that Cullin-3 was required for striated muscle development, a finding that our studies fully support. However, no molecular mechanism that explained some of the observed muscle defects was provided.

We performed in-depth characterization of mice lacking Cullin-3 in skeletal muscles. Specifically, we focused on the role of Cullin-3 for the diaphragm, a hot spot for Cullin-3 substrate adaptor function. Our data show the importance of Cullin-3 for myoblast fusion, differentiation, and neuromuscular junction (NMJ) establishment through the modulation of actin dynamics and the regulation of protein levels for the non-muscle $\alpha$-actinin isoforms ACTN1 and ACTN4. Finally, we also found specific accumulation of ACTN1 and ACTN4 only in the muscles of patients with Cullin-3-related nemaline myopathy, characterized by mutations in KBTBD13. In addition, our study revealed that ACTN1 and ACTN4 are constituents of nemaline bodies in these patients.

\section{Results}

Generation and validation of skeletal muscle Cul3-knockout mice. To explore the role of Cullin-3 during skeletal muscle development, we crossed $C u l 3^{f l f l}$ mice (21) with transgenic animals that express Cre recombinase under the control of the myogenin promoter and the MEF2C enhancer (22) (Supplemental 
A
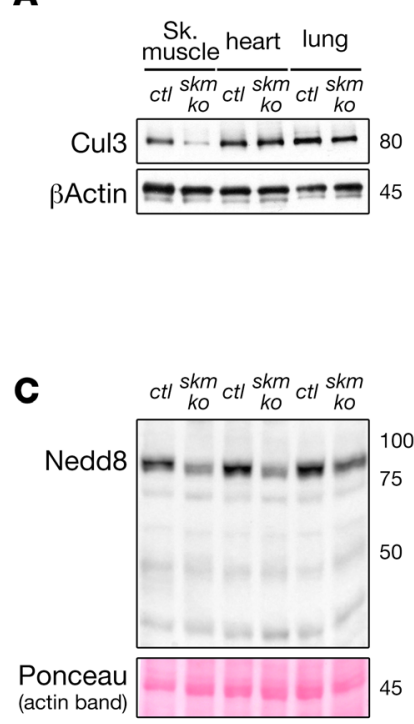

B

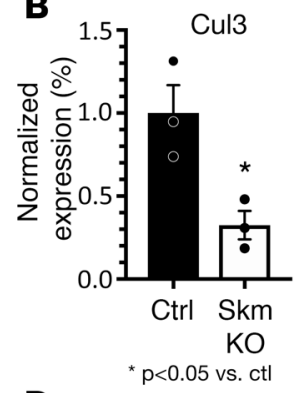

D

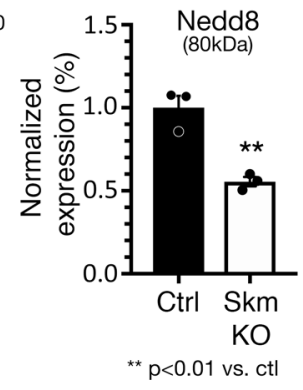

E

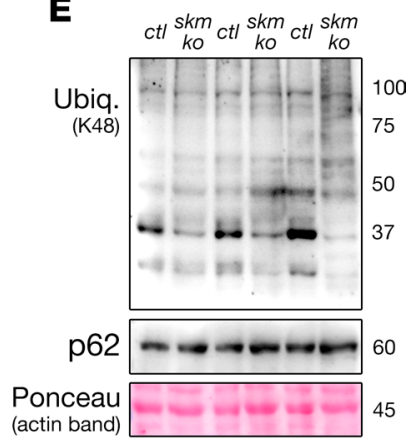

(actin band)

Figure 1. Deletion of Cullin-3 in skeletal muscles affects the ubiquitin-proteasome system. (A) Immunoblot analysis showing loss of Cullin-3 (Cul3) protein only in skeletal muscle (Sk. muscle) of E18.5 skm-KO mice. (B) Quantification of Cullin-3 protein levels in E18.5 skeletal muscles ( $n=3$ for each genotype). ${ }^{*} P<0.05$ by 2 -tailed $t$ test. ctl, control. (C) Immunoblot analysis showing a decrease in NEDD8-associated proteins in E18.5 skeletal muscles of skm-KO mice. (D) Quantification of 80-kDa NEDD8-associated protein levels in E18.5 skeletal muscles ( $n=3$ for each genotype). ${ }^{* *} P<0.01$ by 2-tailed $t$ test. (E) Immunoblot analysis showing a decrease in low molecular weights of K48-ubiquitin-associated proteins [Ubiq. (K48)] and no change in p62 expression levels in skeletal muscles of E18.5 skm-KO mice.

Figure 1, A and B; supplemental material available online with this article; https://doi.org/10.1172/ jci.insight.125665DS1). Transcript and protein levels of Cullin-3 were subsequently monitored by RT-PCR and immunoblot analyses. Efficient recombination of the Cullin-3 gene was observed only in

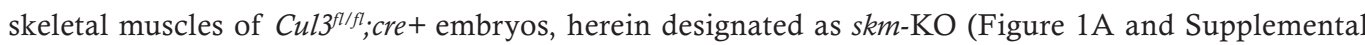
Figure 1C). In diaphragms, protein levels of Cullin- 3 were diminished by $70 \%$ compared with controls (Figure 1, A and B). Remaining Cullin-3 was likely due to its expression in other cell types that are present in skeletal muscles (e.g., blood vessels, neural tissues, or fibroblasts). Analysis of Cullin-3 protein levels in other tissues did not reveal significant changes (Figure 1A). We did not identify changes in mRNA levels of other Cullin family members (Supplemental Figure 1D).

Changes to global protein neddylation was assessed by immunoblot analysis and revealed a $45 \%$ decrease in the $80-\mathrm{kDa}$ band, consistent with the predicted sizes of most Nedd8-modified Cullin proteins $(23,24)$ (Figure 1, C and D). This substantial change suggests that Cullin-3 represents a major constituent of all active Cullins detected in the developing skeletal muscles. Analysis of K48-linked polyubiquitylated substrates, which are normally targeted to the proteasome for degradation, revealed a decrease in low-molecular-weight bands (Figure 1E). Because the UPS and autophagy-lysosome system are interconnected, we assessed p62 protein levels as marker for changes to cellular autophagy. Levels of p62 in skm-KO mice were unchanged compared with controls (Figure 1E), excluding compensatory roles played by the autophagy-lysosome system to overcome deficiency of the ubiquitin-proteasome pathway. In summary, depletion of Cullin-3 in our mouse model was restricted to skeletal muscles and led to a decrease in global neddylation and polyubiquitylation of proteins without affecting p62 expression.

Skeletal muscle expression of Cullin-3 is required for neonatal survival. Genotype analysis of animals at weaning stage (P21) demonstrated complete absence of skm-KO mice (Supplemental Figure 2A). In comparison, $s k m-\mathrm{KO}$ embryos were present at expected Mendelian ratios at E18.5 (Supplemental Figure 2A). While $s k m-\mathrm{KO}$ embryos were able to form skeletal muscles, our analyses revealed a fully penetrant postnatal 
A
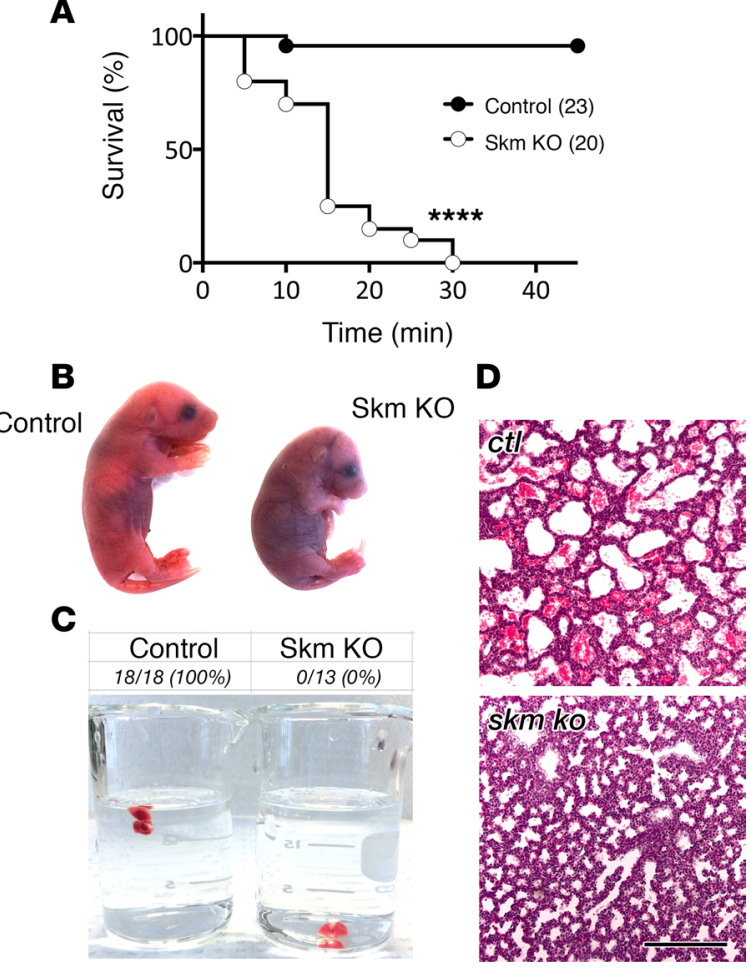

Figure 2. Loss of Cullin-3 during skeletal muscle development leads to postnatal death and respiratory defects. (A) Survival curve of E18.5 embryos following C-section ( $n=23$ for control [ctl] and $n=20$ for skm-KO). ${ }^{* * *} P<0.0001$ by log-rank (Mantel-Cox) and Gehan-Breslow-Wilcoxon tests. (B) Representative pictures of E18.5 embryos showing cyanosis and kyphosis of skm-KO mice. (C) Floating assay using lungs extracted from E18.5 skm-KO embryos and controls 5 minutes after the C-section and placed at the surface of water ( $n=18$ for ctl and $n=13$ for skm-KO). (D) Cross section of E18.5 lungs 5 minutes after C-section stained with $\mathrm{H} \& \mathrm{E}$, revealing collapsed alveoli in skm-KO embryos.

lethality phenotype. Indeed, all skm-KO embryos died within 30 minutes following C-section (Figure 2A). $s k m-\mathrm{KO}$ neonates showed few and slow spontaneous movements, and rapidly turned cyanotic (Figure 2B and Supplemental Figure 2B), which was highly suggestive of respiratory defects. Analysis of their gross anatomy revealed strong spine curvature and wrist drop (Figure 2B), both hallmarks of muscle weakness. To evaluate whether $s k m$-KO embryos were able to breathe, lungs were collected and placed immediately on the surface of water. In contrast to controls, lungs of $s k m-\mathrm{KO}$ embryos sank, indicating that they had never been inflated (Figure 2C). Histological analyses showed that lung alveoli of $s k m$-KO embryos were not expanded (Figure 2D), again indicating that $s k m-\mathrm{KO}$ embryos were unable to breathe. Our data confirm previous reports (20) that expression of Cullin-3 in skeletal muscles is absolutely required for neonatal survival and that the cause of death in $s k m-K O$ mice is due to a breathing defect.

Loss of Cullin-3 leads to severe myopathy characterized by skeletal muscle maturation defects and fiber hypotrophy. Focusing on E18.5 skm-KO embryos, we found a $22.3 \%$ decrease in body weight compared with controls (control $1.35 \pm 0.02 \mathrm{~g}, s k m-\mathrm{KO} 1.05 \pm 0.02 \mathrm{~g} ; P<0.0001$; Figure 3A). However, tibia lengths were not significantly changed (control $1.8 \pm 0.2 \mathrm{~cm}, s k m-K O 1.7 \pm 0.2 \mathrm{~cm} ; n=5$ for each genotype). These data indicate that the decrease in body weight is not due to global prenatal growth retardation but may be more attributable to a $65 \%$ decrease in skeletal muscle mass (Figure 3B and Supplemental Figure 3A). Loss of skeletal muscle was also observable in diaphragm and hind limb cross sections stained with H\&E (Figure 3C and Supplemental Figure 3, B and C). Masson's trichrome did not reveal abnormal fibrosis (data not shown). However, Gomori modified trichrome staining showed the presence of aggregates (Supplemental Figure 3D). This phenotype was reminiscent of observations made in nemaline myopathies associated with mutations in genes encoding for substrate adaptors of Cullin-3 (13).

Because mutations in genes encoding for Cullin-3 substrate adaptors are mainly associated with early-onset myopathies (13), we hypothesized that muscle maturation in the absence of Cullin-3 may be affected. We assessed several sarcomeric proteins, markers of mature muscles, and found a severe decrease in the 
A

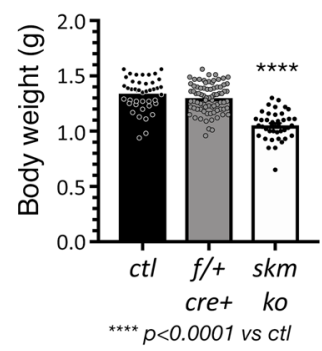

B

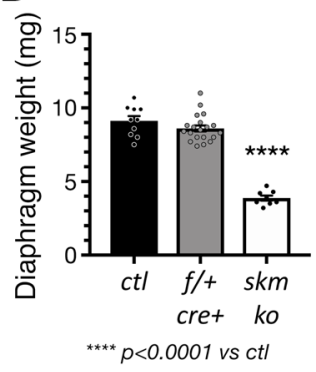

C

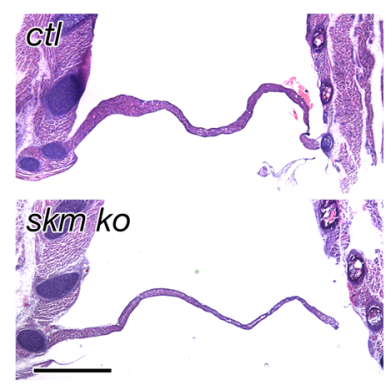

D

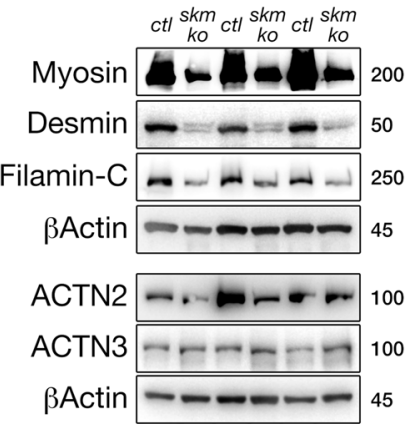

E

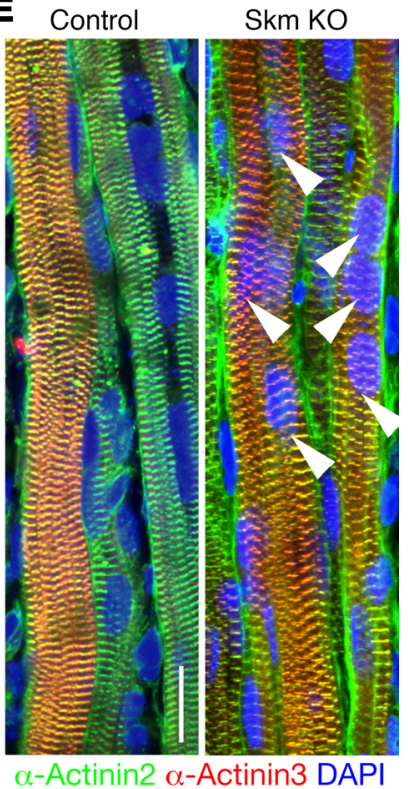

Figure 3. Absence of Cullin-3 leads to severe skeletal muscle myopathy. (A) Body weight analysis of E18.5 embryos ( $n=43$ for $\mathrm{ctl}, n=79$ for heterozygous [ $f /+; \mathrm{cre}+]$, and $n=41$ for $s \mathrm{~km}-\mathrm{KO})$. ${ }^{* * * *} P<0.001$ by ANOVA and Bonferroni's multiple-comparisons test. (B) Diaphragm weight analysis, revealing strong muscle atrophy of E18.5 skm-KO embryos. $n=10$ for ctl, $n=19$ for heterozygous ( $f /+$; cre + ), and $n=8$ for skm-KO. ${ }^{* *} P<0.0001$ by ANOVA and Bonferroni's multiple-comparisons test. (C) Cross section of E18.5 diaphragms stained with $\mathrm{H} \& \mathrm{E}$ showing thinner muscle in skm-KO. Scale bar: $1 \mathrm{~mm}$. (D) Immunoblot analysis showing a decrease in expression of muscle maturation markers in skm-KO diaphragms ( $n=3$ for each genotype). (E) Immunofluorescence staining of diaphragm myofibers with muscle ACTN2 and ACTN3 antibodies as well as DAPI. Arrowheads indicate centralized nuclei. Scale bar: $20 \mu \mathrm{m}$.

expression of sarcomeric myosin heavy chain, desmin, and filamin-C (Figure 3D and Supplemental Figure 4, A-C). We also noticed trends toward decreased expression of sarcomeric $\alpha$-actinin 2 (ACTN2) and increased expression of ACTN3 (Figure 3D and Supplemental Figure 4, D and E) in skm-KO diaphragms. However, none of these changes reached significance. During development, myonuclei first locate to the center of fibers, then migrate to the periphery in more mature muscles (25). In $s k m$-KO embryos, we observed widespread presence of centralized nuclei, while they were mainly found at the periphery in controls (Figure 3E and Figure 4A). Together, these data are suggestive of a muscle maturation defect.

In order to investigate the pathogenic mechanism, we assessed whether the reduced muscle mass relied on hypotrophy (a decrease in the size of the fibers) or hypoplasia (a decrease in the number of fibers). We stained diaphragms of control and skm-KO embryos with wheat germ agglutinin (WGA) in order to delineate individual myofibers and analyze their sizes and numbers. The mean fiber cross-sectional area (CSA) was reduced by $62 \%$ (control $95 \pm 5.06 \mu \mathrm{m}^{2}$, skm-KO $32 \pm 1.51 \mu \mathrm{m}^{2} ; P<0.0001$, Figure $4 \mathrm{~A}$ and Supplemental Figure $4 \mathrm{~F}$ ), and the distribution of fiber CSAs was shifted toward smaller diameters in comparison with controls (Figure 4B). However, the number of fibers constituting the diaphragm was unchanged (control $3076 \pm 230, s k m-K O 2654 \pm 470$; Supplemental Figure 4G), suggesting that fiber hypotrophy accounted for the reduced muscle mass.

It is established that during early steps of muscle development, myoblast fusion represents a limiting step for optimal myofiber size and muscle mass (26-33). We tested whether a defect in myoblast fusion could be responsible for the muscle fiber hypotrophy observed in embryonic skm-KO diaphragms. We isolated myoblasts from E18.5 control and $s k m-\mathrm{KO}$ muscles and validated that $s k m-\mathrm{KO}$ myoblasts undergo recombination of the Cullin-3 locus upon expression of myogenin during differentiation (Figure 4C).

We then assessed myoblast fusion after 3 days of differentiation and observed a defect in skm-KO myotube formation (Figure 4D). skm-KO myoblasts expressed myogenic factors such as myosin heavy chain (MyHC) but did not form long myotubes (Figure 4D), as confirmed by the decrease in their fusion index defined as the number of nuclei per myotube (Supplemental Figure $4 \mathrm{H}$ ), pointing out a fusion defect. 
A
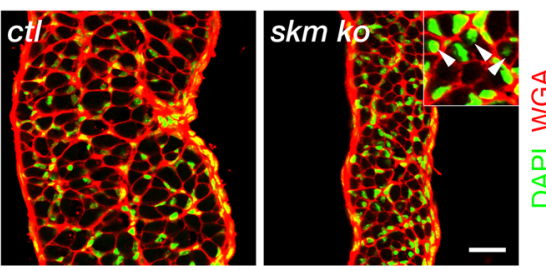

C

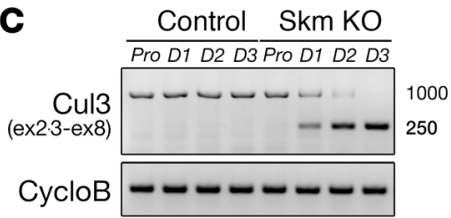

D

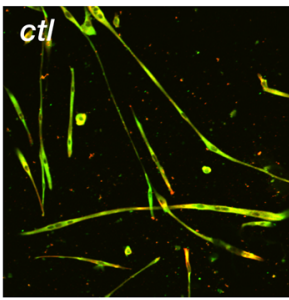

$\mathrm{MyHC}$

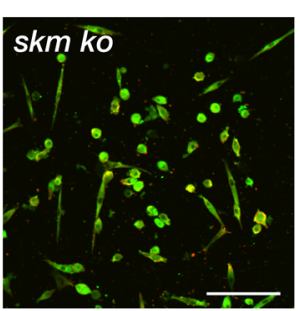

$\beta$-Actin
B

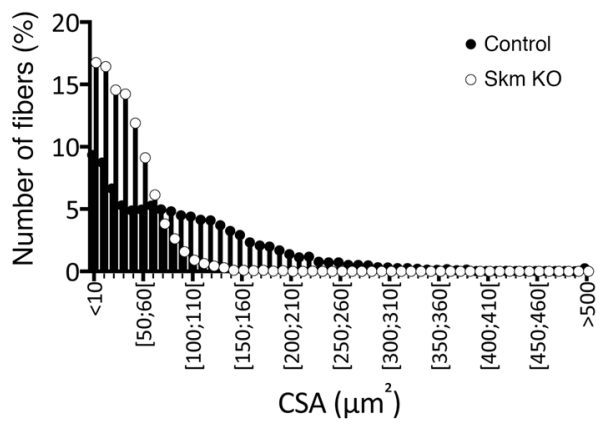

E
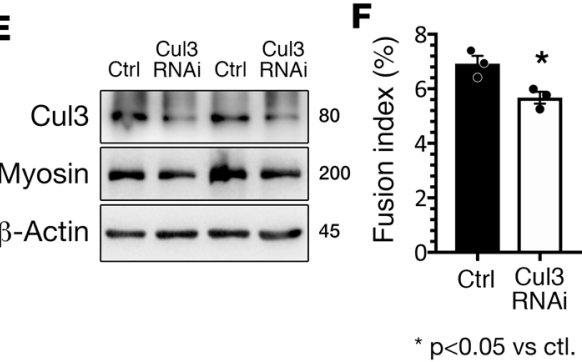

Figure 4. Loss of Cullin-3 leads to muscle fiber hypotrophy in vivo and myoblast fusion defect in vitro. (A) Immunofluorescence staining of E18.5 diaphragms with WCA and DAPI revealing hypotrophy of the myofibers of skm-KO embryos. Arrowheads indicate centralized nuclei. Scale bar: $100 \mu \mathrm{m}$. (B) Distribution of fibers constituting diaphragms of E18.5 ctl and skm-KO depending on their cross-sectional area (CSA in $\left.\mu \mathrm{m}^{2}\right)(n=3$ embryos for each genotype and $>11,554$ fibers per genotype). (C) RT-PCR analysis of Cullin-3 and Cyclophilin B (CycloB) in satellite cells isolated from E18.5 ctl and skm-KO diaphragms over 3 days of differentiation in culture. Pro, proliferation; D1-D3, differentiation days 1-3. (D) Immunofluorescence of satellite cells fixed after 3 days of differentiation and stained with MyHC and $\beta$-actin antibodies. Scale bar: $100 \mu \mathrm{m}$. (E) Immunoblots of Cullin-3, MyHC, and $\beta$-actin on C2C12 myotubes transfected with an siRNA against Cullin-3 or a scrambled siRNA, showing efficient knockdown. (F) Fusion index (number of nuclei per myotube) of C2C12 cells transfected with a Cullin-3 or a scrambled siRNA and differentiated for 5 days ( $n=3$ per condition and $>144$ myotubes analyzed per experiment). ${ }^{*} P<0.05$ by 2 -tailed $t$ test.

To confirm these results, we transfected C2C12 cells with siRNA against Cullin-3 and monitored their differentiation. We found a $72 \%$ decrease in Cullin-3 protein levels in cells expressing the siRNA compared with cells expressing scrambled siRNA (Figure 4E and Supplemental Figure 4I). We then assessed myoblast fusion 5 days after differentiation and observed a $20 \%$ decrease in the fusion index (Figure $4 \mathrm{~F}$ and Supplemental Figure $4 \mathrm{~J}$ ). While muscle proteins such as MyHC were not significantly changed in the absence of Cullin-3, they trended toward lower expression levels (Figure 4E and Supplemental Figure 4I).

In summary, our data indicate that lack of Cullin-3 impedes a step required for the fusion of myoblasts and maturation of muscle fibers.

Absence of Cullin-3 affects proteins involved in degradation, calcium signaling, metabolism, muscle contraction, and actin modulation. Next, we aimed at identifying molecular mechanisms underlying the muscle defects observed in skm-KO embryos in order to better characterize Cullin-3 functions during skeletal muscle development. While Cullin proteins such as Cullin-1 and Cullin-3 were broadly expressed in various adult skeletal muscles, diaphragm was a hot spot for BTB domain proteins (Cullin-3 substrate adaptors) including KBTBD5, KBTBD13, KLHL9, KCTD6, and PLZF, with the exception of KCTD9 (Figure 5A). To maximize the chances for identifying substrates of Cullin-3 and changes to proteins that may be responsible for the observed phenotype, we performed an unbiased large-scale proteome analysis using E18.5 diaphragms. Our analysis revealed that $6 \%$ of detected proteins were significantly altered in the absence of Cullin-3 (Supplemental Figure 5A). Among those, 26 proteins were significantly accumulated in $s k m-\mathrm{KO}$ diaphragms, while 100 were significantly downregulated (Figure 5B).

Functional analyses aided by DAVID (https://david.ncifcrf.gov) and Metascape (http://metascape.org/) revealed that deregulated proteins might be grouped into 5 categories: heat shock proteins and degradation 
A

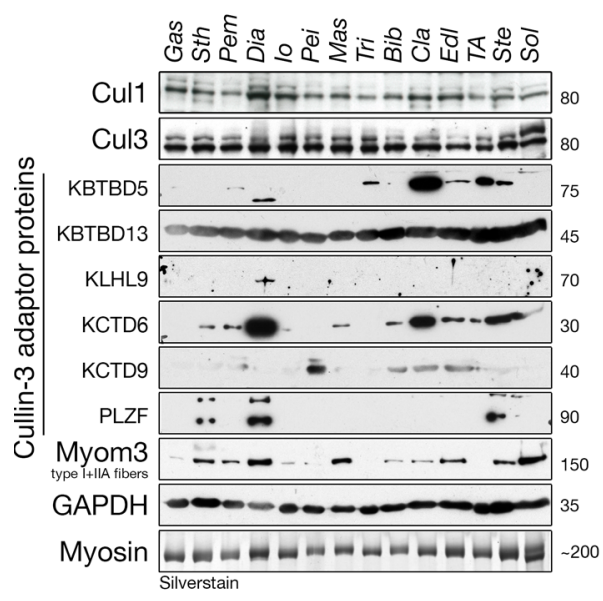

B Upregulated in Downregulated in - Actin modulation (14)

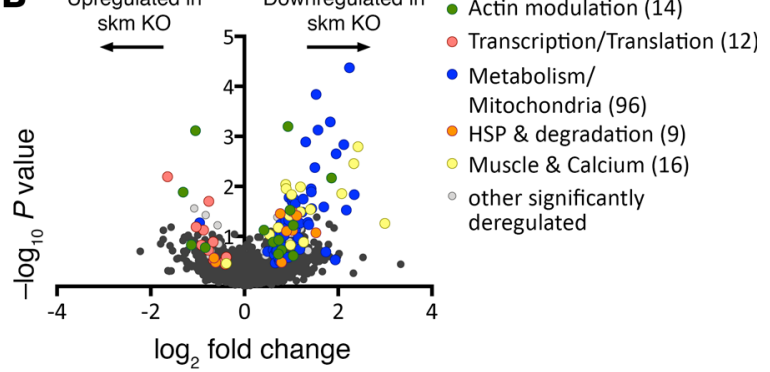

C

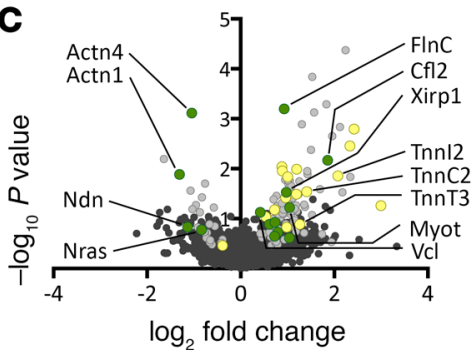

Figure 5. Diaphragm muscle is a hot spot for Cullin-3 substrate adaptors, and depletion of Cullin-3 leads to deregulation of protein levels. (A) Immunoblot analysis showing the pattern of expression of Cullin-1, Cullin-3, and select Cullin-3 substrate adaptors in various mouse skeletal muscles. Myomesin-3 (Myom3) (86) was used as fiber-type marker. Gas, gastrocnemius; Sth, sternohyoideus; Pem, pectoralis major; Dia, diaphragm; lo, internal oblique; Pei, pectoralis minor; Mas, massester; Tri, triceps, Bib, biceps brachii; Cla, clavotrapezius; Edl, extensor digitorum longus; TA, tibialis anterior; Ste, sternomastoideus; Sol, soleus. (B) Volcano plot of all identified deregulated proteins in E18.5 diaphragms of skm-KO mice following ITRAQ mass spectrometry (3 diaphragms were pooled per sample, and 3 samples for each genotype were analyzed). (C) Volcano plot highlighting deregulated proteins involved in actin cytoskeleton modulation as well as proteins related to muscle and calcium handling ( 3 diaphragms were pooled per sample, and 3 samples for each genotype were analyzed).

pathways; muscle- and calcium-associated proteins; proteins associated with metabolism and mitochondria, ribonucleoproteins and DNA-associated proteins; and proteins involved in actin modulation (Figure 5B and Supplemental Figure 5, B-D). Of note, KLHL41 and KLHL31, 2 substrate adaptors involved in muscle physiology $(15,34,35)$, were strongly decreased in skm-KO muscles (Supplemental Figure 5D), suggesting a destabilization of these proteins in the absence of Cullin-3. Altered proteins involved in muscle and calcium physiology, metabolism and mitochondria, HSP, and degradation pathways were mainly downregulated, while ribonucleoproteins and DNA-associated proteins were accumulated. To validate our proteome analysis, we studied the expression of mitochondrial subunits by immunoblot analysis using the OxPhos antibody cocktail. A strong decrease in almost all mitochondrial complex subunits was confirmed in $s k m$-KO diaphragms (Supplemental Figure 5C), validating our proteome analysis.

Cullin-3-knockout muscle cells accumulate non-muscle isoforms of $\alpha$-actinin. A surprising result was the strong deregulation of proteins involved in actin cytoskeleton modulation. Actin-binding proteins were found both accumulated and downregulated in $s k m-\mathrm{KO}$ diaphragms (Figure 5, B and C). Because of the pivotal role that remodeling of the actin cytoskeleton plays in muscle development, including myoblast fusion $(36,37)$, we decided to focus on these proteins.

Among them, non-muscle ACTN1 and ACTN4 isoforms were the most significantly accumulated. ACTN4's role in skeletal muscles has been investigated. Surprisingly, ACTN4 overexpression seemed to enhance myoblast differentiation (38). Hence, we focused on the role that ACTN1 may play in our phenotype.

We first confirmed the accumulation of ACTN1 in knockout muscles (Figure 6, A and B) using an antibody that has been validated on muscle cells transfected with siRNA against ACTN1 (Supplemental Figure 6A). In skm-KO myoblasts, we also observed accumulation of ACTN1 during differentiation (Figure 6, C and D), suggesting that accumulation of ACTN1 in the skeletal muscles of Cullin-3 knockouts originated from muscle cells, and not from other non-muscle tissues. Intriguingly, we observed a similar result in muscles of HSA-Cre inducible Cullin-3-knockout (iCul3-KO) mice. Loss of Cullin-3 in adult muscles 1 month after excision of the gene by doxycycline treatment resulted in the accumulation of ACTN1, while sarcomeric ACTN2 remained unchanged (Figure 6, E and F). Together, our data indicate that Cullin-3 ablation in muscle cells is responsible for the accumulation of ACTN1. 

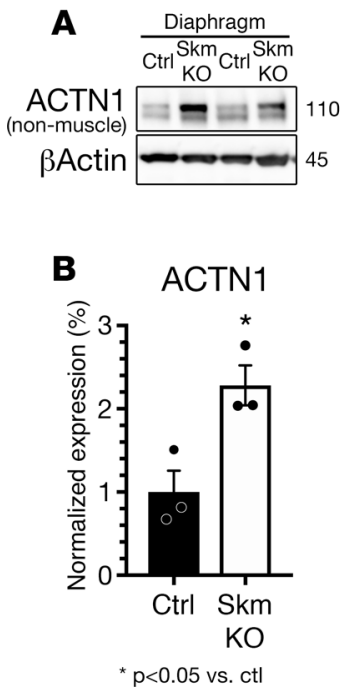

C
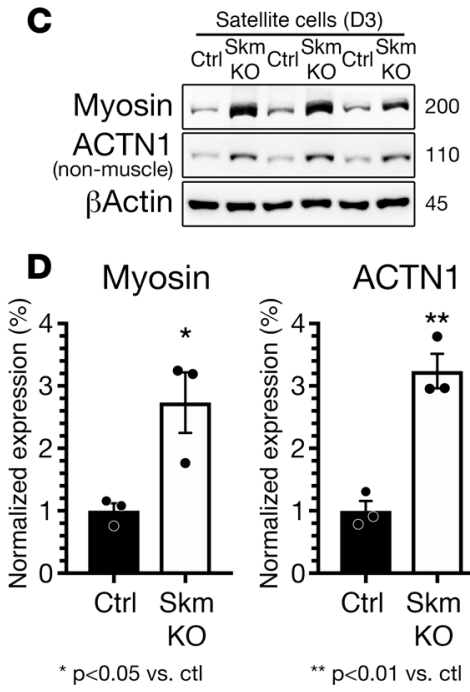

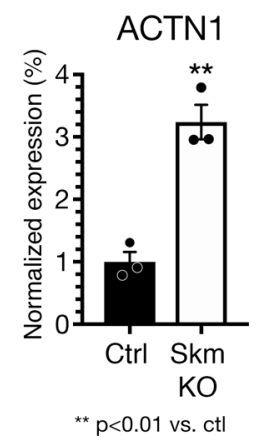

$\mathbf{E}$

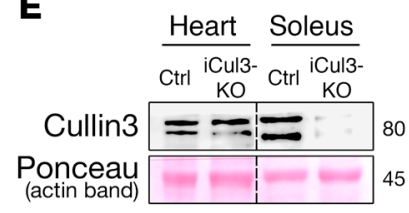

$\mathbf{F}$

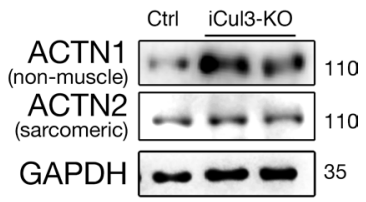

Figure 6. Loss of Cullin-3 induces accumulation of ACTN1. (A) Immunoblot analysis showing accumulation of ACTN1 in diaphragms of skm-KO embryos. (B) Quantification of ACTN1 protein levels in E18.5 diaphragms of ctl and skmKO mice ( $n=3$ embryos for each genotype). ${ }^{*} P<0.05$ by 2 -tailed $t$ test. (C) Immunoblots showing accumulation of ACTN1 and deregulation of sarcomeric myosin in satellite cells of $s \mathrm{~km}-\mathrm{KO}$ embryos after 3 days of differentiation. (D) Quantification of ACTN1 protein and myosin levels in satellite cells of ctl and $s k m-K O$ embryos after 3 days of differentiation. $n=3$ technical replicates for each genotype. ${ }^{*} P<0.05,{ }^{* *} P<0.01$ by 2 -tailed $t$ test. (E) Immunoblot analysis showing the specific loss of Cullin-3 in skeletal muscles of iCul3-KO (doxycycline-inducible Cullin-3-knockout) mice. Dotted line indicates that samples for hearts and soleus have been run on the same gel but not in adjacent lanes. (F) Immunoblot analysis showing accumulation of ACTN1, but not sarcomeric ACTN2, in skeletal muscles of iCul3-KO mice treated with doxycycline.

We then investigated the role that ACTN1 may play during early muscle differentiation. First, we monitored the expression of ACTN1 at the RNA and protein levels during C2C12 differentiation. While sarcomeric ACTN2 increased during differentiation, we found that ACTN1 and ACTN4 were downregulated (Supplemental Figure 6, B and C). However, RNA levels of Actn1 remained unchanged during differentiation (Supplemental Figure 6D). This result suggested active regulation of ACTN1 protein levels and a role for Cullin-3 during muscle cell differentiation. In proliferative $\mathrm{C} 2 \mathrm{C} 12$ cells, ACTN1 localized at the edge of the cells and along the actin stress fibers and focal contacts, confirming previous data in other cell types (39) (Supplemental Figure 6E). Three days after differentiation, when myoblast fusion occurs, ACTN1 staining became more fragmented in a subset of cells. In other cells, it retained its localization along actin stress fibers and focal adhesion contacts (Supplemental Figure 6E). Finally, 5 days after differentiation, while ACTN1 still localized at the edge of mononucleated and nonfused cells, it was weakly expressed and diffusely localized in the cytoplasm of myotubes. Together, these data suggest very active and intense remodeling of ACTN1 during myogenesis and myoblast fusion.

Overexpression of ACTN1 in differentiating myoblasts leads to fusion defects. To test our hypothesis of a causal role for the abnormal accumulation of ACTN1 and the pathological modulation of actin cytoskeleton proteins in the phenotype of skm-KO embryos, we generated a $\mathrm{C} 2 \mathrm{C} 12$ cell line that stably overexpresses HA-ACTN1. We first validated the correct expression of HA-ACTN1 in C2C12 cells (Figure 7A and Supplemental Figure 7A) and monitored their differentiation. Five days after differentiation, HA-ACTN1overexpressing cells displayed a reduction in the ability to form myotubes compared with control cells (Figure 7B), accompanied by a slight but consistent decrease in MyHC expression (Figure 7A and Supplemental Figure 7B). Our analyses indicated a 44\% decrease in the fusion index of HA-ACTN1 cells compared with controls (Figure 7C) due to an overrepresentation of small myotubes (2-4 nuclei), and an almost complete loss of myotubes containing larger amounts of nuclei ( $\geq 54$ nuclei) (Figure 7D).

Together our data suggest that accumulation of ACTN1 impaired the fusion of differentiating myoblasts, resulting in smaller myotubes. These findings were reminiscent of the phenotype of muscle fibers and satellite cells from Cullin-3-knockout animals, suggesting that accumulation of ACTN1 is responsible for at least part of the phenotype observed in Cullin-3-depleted tissues. 
A

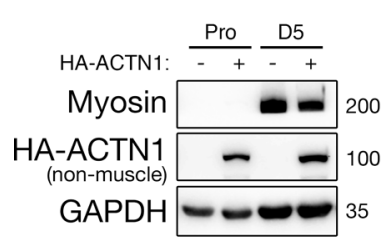

C

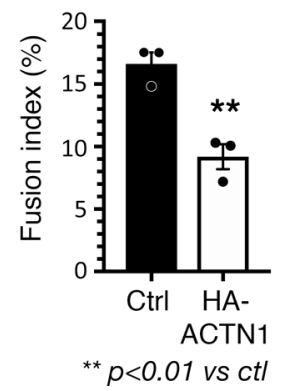

B
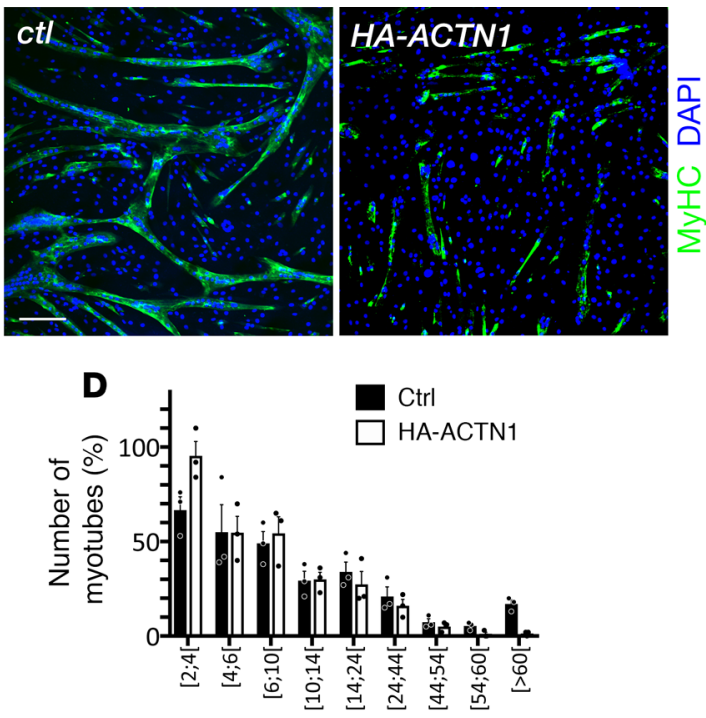

Nuclei/myotube

Figure 7. Overexpression of ACTN1 in muscle cells leads to differentiation defects. (A) Immunoblot analysis showing overexpression of ACTN1 in C2C12 cells overexpressing the HA-ACTN1 construct. Pro, proliferation; D5, differentiation day 5. (B) Immunofluorescence staining of HA- (ctl) and HA-ACTN1-overexpressing myotubes with sarcomeric myosin antibody and DAPI after 5 days of differentiation. Scale bar: $200 \mu \mathrm{m}$. (C) Fusion index of cells expressing HA (ctI) and HA-ACTN1 constructs 5 days after differentiation, showing a decrease in the number of nuclei per myotube ( $n=3$ for each condition). ${ }^{* *} P<0.01$ by 2-tailed $t$ test. (D) Distribution of myotubes depending on the number of nuclei ( $n=3$ for each condition).

CRL activity is increased during acetylcholine receptor clustering and leads to the increase in polyubiquitylated proteins. Impairment of NMJ development often results in respiratory defects due to the dysfunction of the diaphragm and intercostal muscles (40). Importantly, a striking sustained and beneficial effect of acetylcholine esterase inhibitor treatment has been observed in a patient affected by KLHL40-related nemaline myopathy, another confirmed substrate adaptor for Cullin-3 (41). In addition, sarcomeric ACTN2 has been shown to interact with rapsyn (42), and active actin cytoskeleton remodeling is absolutely required for proper acetylcholine receptor (AchR) clustering and NMJ development (43).

Cullin E3-ligase activity is required for normal AchR clustering in vitro $(11,44)$. We first monitored Cullin-3 expression following stimulation of C2C12 myotubes with neural agrin. We observed that Cullin-3 was significantly increased upon AchR clustering (Figure 8A and Supplemental Figure 8A), suggesting a role during NMJ development. We then looked at the expression pattern of Nedd8, the marker for Cullin activity, during AchR clustering. Following 48 hours of agrin stimulation, we found a strong increase in Nedd8 levels at $80 \mathrm{kDa}$ (Figure 8B and Supplemental Figure 8B), suggesting an increase in Cullin E3 ligase activity during this process. We also observed a significant increase in polyubiquitylated proteins (Figure $8 \mathrm{C}$ and Supplemental Figure $8 \mathrm{C}$ ), indicating an increase in protein degradation during AchR clustering. In order to test whether the increase in polyubiquitylation was due to the increase in Cullin E3 ligase activity, we treated myotubes with both agrin and MLN4924 (a CRL inhibitor). In the absence of Cullin activity, as shown by the loss of Nedd8 signal around $80 \mathrm{kDa}$ (Figure $8 \mathrm{D}$, left panel), the global increase in polyubiquitylation observed in control conditions was inhibited (Figure $8 \mathrm{D}$, right panel), demonstrating a causal role for CRLs in the increase in polyubiquitylation during AchR clustering. Together, these data indicate that Cullin-3 is increased during AchR clustering in vitro and that increased Cullin activity is in part responsible for protein degradation during AchR clustering.

Following these results, we decided to assess NMJs in vivo, specifically focusing on organization and AchR clustering in skm-KO diaphragms. We performed immunofluorescence analyses of post- and presynaptic elements. Our data revealed complete disorganization of the NMJs in muscle lacking Cullin-3 (Figure 9A). While AchR clusters were aligned along a narrow band in the center of embryonic control diaphragms, they appeared scattered in $s k m-K O$ diaphragms (Figure 9A). In addition, staining of synaptophysin/neurofilament indicated abnormal branching and arborization of the motoneuron (Figure 9, A and B), likely due to the mislocalization of the AchR clusters. Quantification showed an almost 4-times 


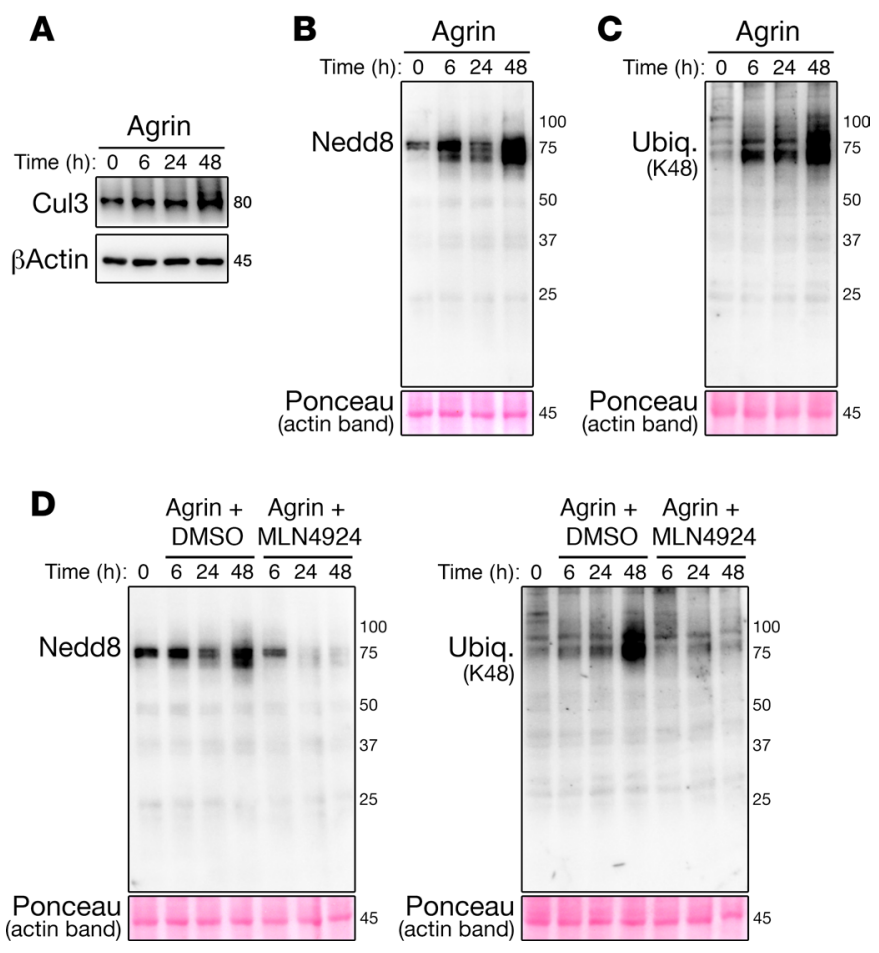

Figure 8. Cullin-3 protein levels and Cullin-RING ligase activity are increased over AchR clustering in vitro. (A) Immunoblot analysis showing increased expression of Cullin-3 protein levels in C2C12 cells stimulated with neural agrin $(0.5 \mu \mathrm{g} / \mathrm{ml})$ for 48 hours in order to trigger acetylcholine receptor (AchR) clustering. (B) Immunoblot analysis showing increased neddylated proteins in C2C12 cells stimulated with agrin for 48 hours. (C) Immunoblot analysis showing increased levels of polyubiquitylated proteins (K48-linked) in C2C12 myotubes stimulated with agrin for 48 hours. (D) Immunoblot analyses showing a decrease in both Nedd8-linked and polyubiquitylated proteins in C2C12 myotubes stimulated with agrin and treated with MLN4924 compared with DMSO.

increase in motor endplate width in skm-KO diaphragms, without an alteration in the percentage of innervated AchRs clusters (Figure 9C). These data suggest that the motoneuron, while hyperarborized and branched, was able to connect with the postsynaptic element during muscle development. We also noticed that AchR clusters were smaller in diaphragms of $s k m-\mathrm{KO}$ embryos compared with controls (Figure 9D) due to an overrepresentation of small clusters and the absence of large ones.

Because we showed previously that loss of Cullin-3 leads to an accumulation of ACTN1 in muscles, we assessed whether ACTN1 overexpression could be responsible for the NMJ phenotype. We differentiated C2C12 cells overexpressing HA-ACTN1 or HA for 5 days, followed by agrin-induced AchR clustering. Analysis of cluster size distribution showed a shift toward accumulation of small clusters and an absence of large ones in HA-ACTN1-expressing cells (Figure 9E), which is reminiscent of the observations made in the diaphragms of $s k m$-KO embryos. In addition, a $21 \%$ decrease in average AchR cluster length was found in myotubes expressing HA-ACTN1 (Supplemental Figure 8, D and E).

Together, these data suggest that Cullin-3 is required for normal NMJ development and that accumulation of ACTN1 affects the formation of AchR clusters.

Non-muscle $\alpha$-actinins are specifically accumulated in muscles of Cullin-3-related nemaline myopathy patients. Mutations in several substrate adaptors for Cullin-3 (i.e., KBTBD13, KLHL9, KLHL40, KLHL41) are associated with the development of distal and nemaline myopathies (13-17). In order to test the relevance of our findings for human health, we analyzed muscle samples from nemaline myopathy patients with mutations in the Cullin-3 substrate adaptor KBTBD13 (16), TPM2 [Chr9(GRCh37):g.35685480A>G, NM_003289.3(TPM2):c.443T>C], or $N E B$ [chr2:152389953T>C, c.21522+3A>G (NM_001271208.1); chr2:152581433delG, c.445delC (NM_001271208.1)]. Mutations in KBTBD13 are mainly associated with early-onset nemaline myopathies, characterized by muscle weakness, slow movement, pronounced muscle fiber atrophy, and the presence of nemaline bodies (16). KBTBD13 is a substrate adaptor for Cullin-3 (45). We confirmed the presence of nemaline bodies in our patients with mutations in KBTBD13 through 
A
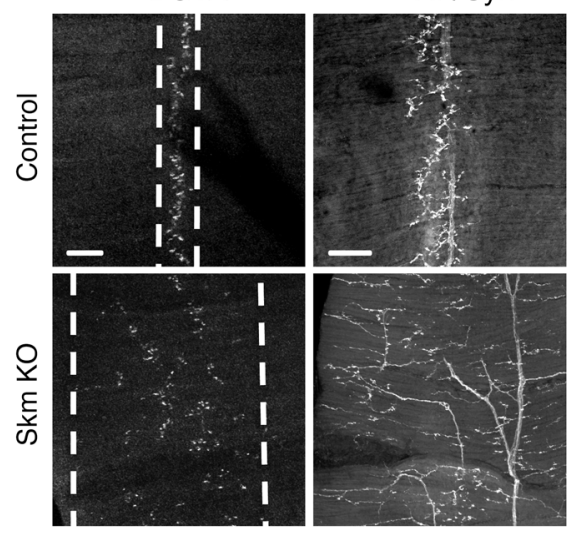

B

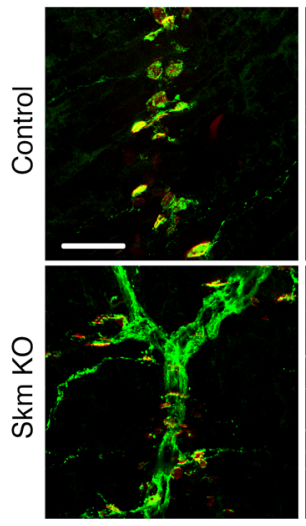

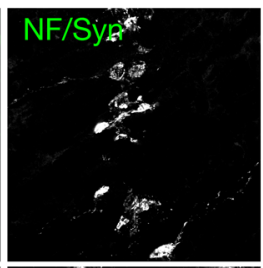
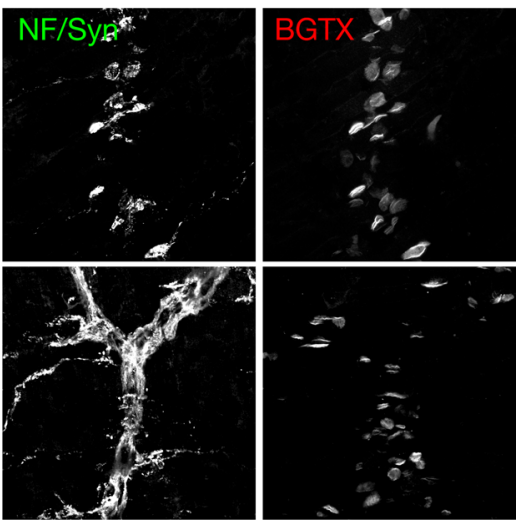

C

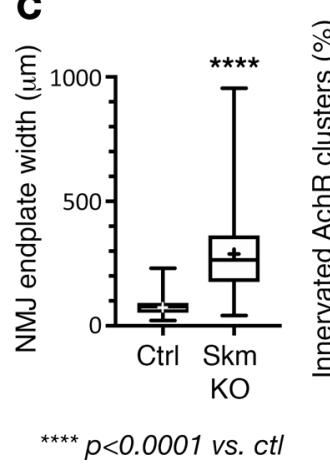

D

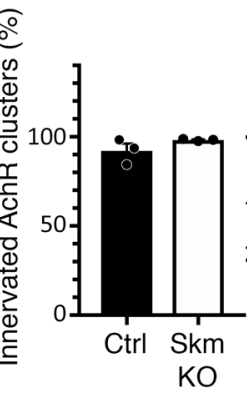

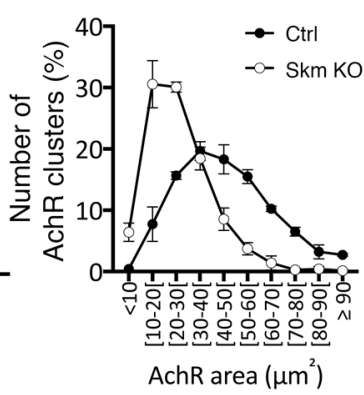

E

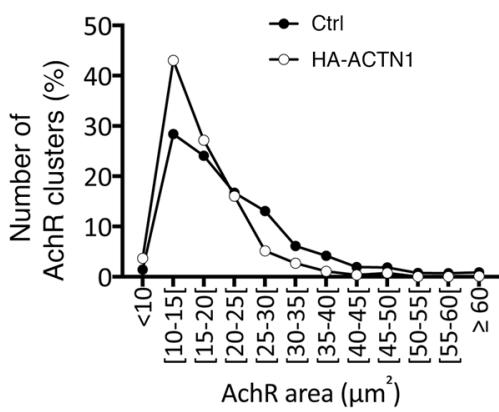

Figure 9. Cullin-3 is required for normal neuromuscular junction formation and acetylcholine receptor clustering. (A and B) Immunofluorescence staining of the pre- and postsynaptic elements of E18.5 neuromuscular junctions (NMJs) in ctl and skm-KO diaphragms with antibodies against neurofilament and synaptophysin (NF/Syn) and fluorescent bungarotoxin (BGTX), showing (A) increased area of the motor endplate, dispersion of acetylcholine receptor (AchR) clusters across the diaphragm, and hyperarborization of the motoneuron in skm-KO embryos; but (B) normal innervation of the NMJ. Scale bars: 100 (A) $\mu \mathrm{m} ; 20 \mu \mathrm{m}$ (B). (C) Quantification of NMJ endplate widths (left panel; box-and-whiskers plot showing minimum to maximum values; + indicates average) and innervated AchRs (right panel) in the diaphragms of ctl and skm-KO embryos ( $n=3$ embryos for each genotype, $>895$ AchRs per genotype). ${ }^{* * *} P<0.0001$, by 2-tailed $t$ test (left panel); $P=0.211$ by 2-tailed $t$ test (right panel). (D) Distribution of AchR clusters of ctl and skm-KO diaphragms according to their area ( $n=3$ embryos for each genotype). (E) Distribution of AchR cluster areas in 5-day-differentiated myotubes expressing HA (ctl) or HA-ACTN1 ( $n=3$ per condition and $>817$ AchR clusters were analyzed per condition).

Gomori trichrome staining (Figure 10A). We then investigated protein levels of actinin isoforms. While levels of ACTN2 and ACTN3 were mainly unchanged across the patients and controls, we found a striking increase in ACTN1 and ACTN4 levels only in KBTBD13 patients (Figure 10B), mimicking results obtained in the muscles of $s k m$-KO embryos. Finally, we asked whether nemaline bodies, features shared by all nemaline myopathy patients, are positive for non-muscle $\alpha$-actinin isoforms. While, nemaline bodies are known to be positive for ACTN2, we found that they were also reactive to ACTN1 and ACTN4 in muscle biopsies of KBTBD13 patients (Figure 10C).

Together, these data reveal an abnormal accumulation of non-muscle ACTN1 and ACTN4 in nemaline myopathy patients with mutations in $K B T B D 13$, which encodes for a Cullin-3-related protein. In addition, we unravel that ACTN1 and ACTN4 accumulate in nemaline bodies of these patients, lengthening the list of proteins constituting these structures.

\section{Discussion}

It has been recently shown that mice lacking Cullin-3 develop severe muscle atrophy and cardiomyopathy, but the responsible molecular mechanism has not been identified (20).

While Nedd8, the crucial regulator for CRL activity, is largely downregulated in a lot of mature tissues, its expression remains high in differentiated muscles (i.e., heart and skeletal muscles) (24), suggesting important roles for CRLs in these tissues. Our data show for the first time to our knowledge that substrate adaptors of Cullin-3 display diverse expression patterns in various skeletal muscle types, with diaphragm 
A

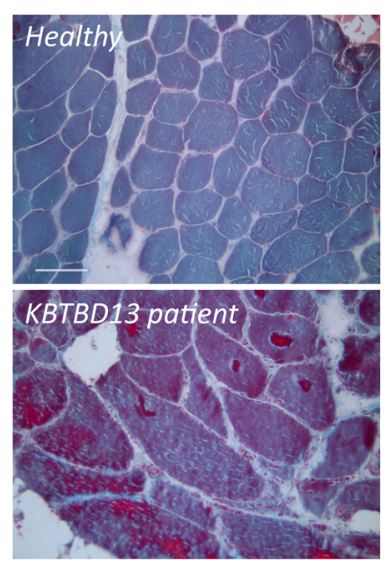

B

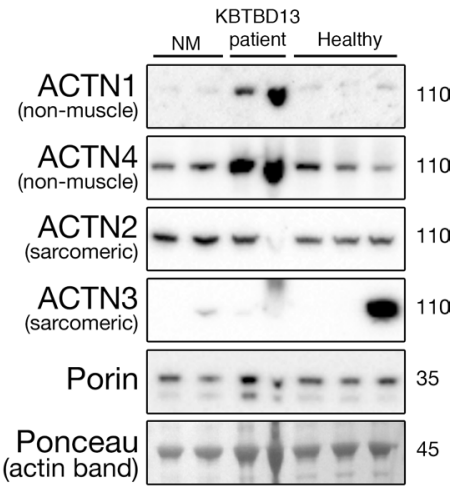

C

는

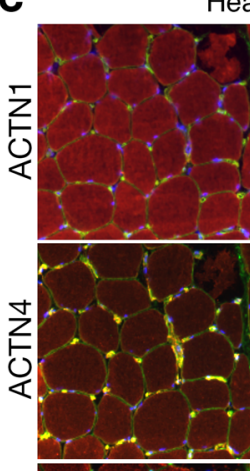

Healthy
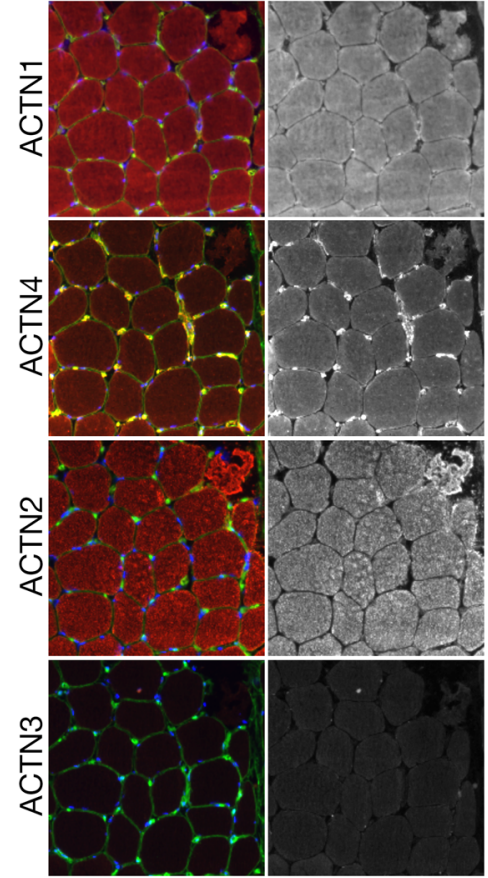

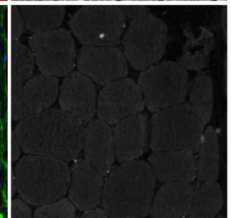

ACTN WGA Hoechst
KBTBD13 patient

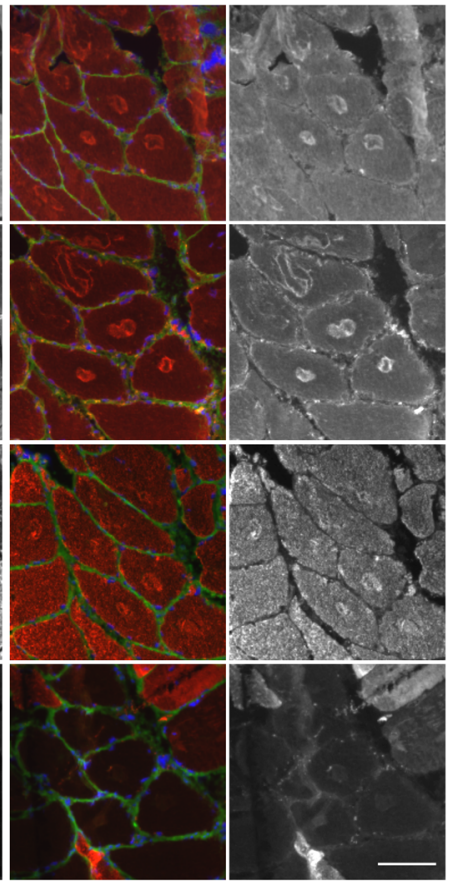

Figure 10. Specific accumulation of non-muscle $\alpha$-actinins in muscle tissues of patients with Cullin-3-related nemaline myopathies. (A) Gomori trichrome staining of skeletal muscle cross sections from a healthy individual and a patient with a mutation in KBTBD13. (B) Immunoblot analyses of $\alpha$-actinin isoforms expression levels in skeletal muscle biopsies from nemaline patients (NM patients; lane 1: TPM2 mutation; lane 2: NEB mutation), patients with mutations in KBTBD13 (lanes 3 and 4), and healthy individuals (lanes 5 and 6). Specific accumulation of non-muscle $\alpha$-actinins (ACTN1 and ACTN4) was observed in samples from patients with mutations in KBTBD13. Muscle $\alpha$-actinins (ACTN2 and ACTN3) did not show abnormal accumulation. Porin and Ponceau stains are shown as loading controls. (C) Immunofluorescence staining of cross sections from muscle biopsies of a healthy individual and a patient with a mutation in KBTBD13, revealing positive staining of nemaline bodies with non-muscle $\alpha$-actinins (ACTN1 and ACTN4). Scale bars: $100 \mu \mathrm{m}$ (A and $\mathbf{C})$.

muscles emerging as a hot spot for their expression. Absence of Cullin-3 impairs the UPS, and is characterized by a decrease in protein neddylation and polyubiquitylation, in differentiating skeletal muscles. These findings suggest that Cullin-3 is a major active CRL during muscle development.

Cullin-3 skm-KO mice exhibit severe hypotrophy of myofibers and die within a few minutes after birth. Cullin-3-depleted myoblasts ( $\mathrm{skm}$-KO satellite cells and $\mathrm{C} 2 \mathrm{C} 12$ cells treated with Cullin-3 siRNA), while able to express myogenic factors, were unable to form large myotubes, suggestive of impaired myoblast fusion. Comparisons with mouse models exhibiting a similar phenotype indicate 
Control muscle

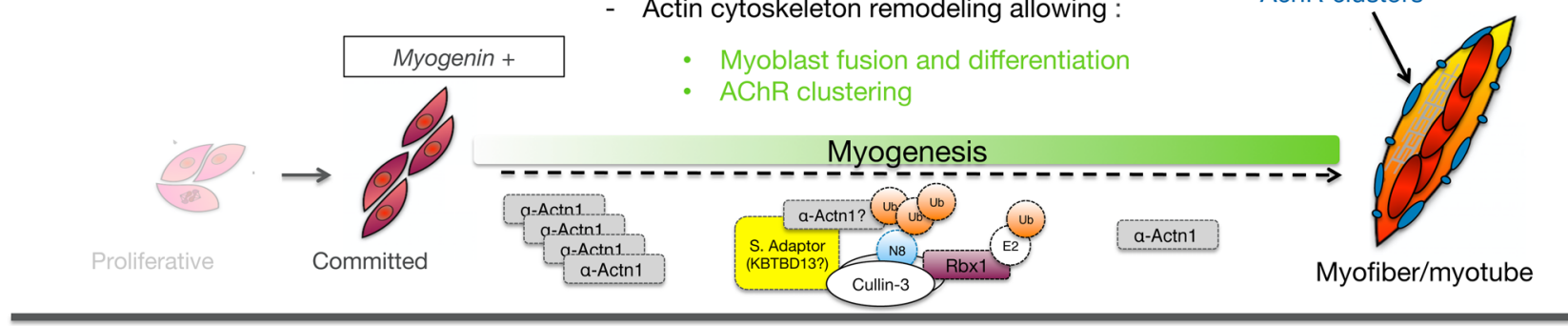

Cullin-3 skm-KO, Cullin-3 siRNA, KBTBD13 mutation or a-Actn1 overexpression

- Accumulation of a-Actn1

- Defect in actin cytoskeleton remodeling

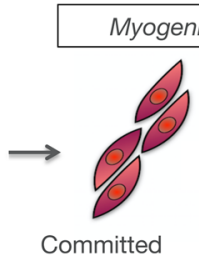

- Fusion defect (hypotrophy of muscle)

- AChR clustering defect (smaller and disorganized)

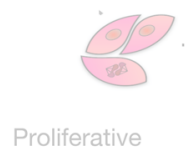

Committed
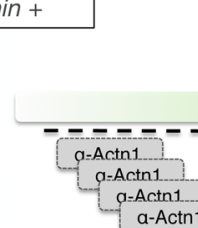

a-Actn1
Myogenesis

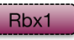

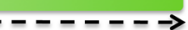

a-Actn1

a-Actn1

a-Actn1
AchR clustering defect

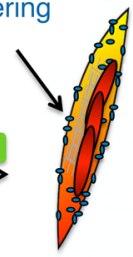

Fiber hypotrophy

Absence or deregulation of Cullin-3 and/or a-Actn1 overexpression

Figure 11. Model for the role of Cullin-3 during muscle fiber development. S. Adaptor, substrate adaptor; Rbx1, RING-box protein 1; AchR, acetylcholine receptor; Ub, Ubiquitin; N8, Nedd8.

that muscle hypotrophy and fusion defects may be at least partially responsible for the death of Cullin-3 skm-KO mice (26-33).

Intriguingly, skm-KO embryos share a similar phenotype with some knockout models for NMJ components, such as Agrin, Choline acetyltransferase, MuSK, or Rapsyn (40). The models are characterized by rapid postnatal lethality due to respiratory defects resulting from insufficient muscle development and improper transmission of neuronal signals to the muscle (40). Due to the striking beneficial effects of acetylcholine esterase inhibitor treatment in a KLHL40-related nemaline myopathy patient (41), we asked whether Cullin-3 could also be required for NMJ formation and function. Our data uncovered a function for Cullin-3 during development of NMJs, an essential process for myogenesis and muscle growth (40). We demonstrated that Cullin-3 and CRL activity increases during AchR clustering and is required for the increased level of polyubiquitylated proteins occurring during this process. Increasing evidence suggests that protein ubiquitylation may play an important role in regulating synapse development (46). Interestingly, it was demonstrated that RPY-1, the homolog of rapsyn in Caenorhabditis elegans, can be degraded by Cullin-3 $(47,48)$. While those findings link Cullin-3 with NMJ development in nematodes, this mechanism has not been further investigated in mammals.

In this study, we found that loss of Cullin-3 in vivo and in vitro led to disorganized and smaller AchR clusters, resulting in hyperarborization of the motoneuron. Of note, Cullin- 5 and -2 have already been linked to the development of NMJs in Drosophila melanogaster, but their roles have also not been further corroborated in mammals (49). Together, these data may uncover a more universal function for CRLs during NMJ development.

On the molecular level, our proteome analysis of diaphragm muscles revealed the deregulation of proteins involved in muscle metabolism and mitochondria, calcium handling, protein degradation, actin cytoskeleton modulation, as well as ribonucleosome and DNA-associated proteins. Our proteome analysis diverges from the recently published Cullin-3-knockout model (20). An explanation for this discrepancy is that the authors performed proteome analysis of whole hind limb muscles, while we specifically isolated and investigated the diaphragm. We chose to use diaphragm over hind limb muscles for several reasons: (i) The diaphragm is a hot spot for the expression of Cullin-3 substrate adaptors, increasing the chances to identify molecular mechanisms played by Cullin-3 in skeletal muscles. (ii) In contrast to hind limbs, contamination by other tissue types is reduced by the use and analysis of diaphragm muscles, minimizing the risks of identifying changes that are actually due to non-muscle cells, such as fibroblasts from connective tissue, adipocytes, or endothelial cells. 
The deregulation of non-muscle ACTN1 and ACTN4, vinculin, filamin-C, cofilin-2, myotilin, or Xirp1 triggered our attention. Cullin-3 and BTB domain-containing proteins have already been shown to act as organizers of the cytoskeleton in Drosophila melanogaster $(50,51)$. The remodeling of the actin cytoskeleton is an essential step for efficient myoblast fusion (37) and for AchR clustering at the NMJ (52, 53). While vinculin, filamin-C, cofilin-2, and myotilin have been associated with myoblast fusion and differentiation (54-57) and NMJ $(58,59)$, little is known about the role of ACTN1 in muscles.

The family of $\alpha$-actinin proteins includes the muscle-specific ACTN2 and ACTN3, and 2 non-muscle isoforms, ACTN1 and ACTN4. $\alpha$-Actinins are essential for the stability and architecture of the cytoskeleton, and mutations in these genes lead to pathologies in humans (60-62). ACTN1 and ACTN4 crosslink actin filaments in the cytoskeleton of non-muscle cells, while sarcomeric ACTN2 and ACTN3 anchor the thin filaments and titin at the Z-discs of mature muscles (39). $\alpha$-Actinins form homo- or heterodimers, and each dimer binds to common or specific ligands $(63,64)$, suggesting specific biological roles. In this study, we show that during normal myoblast differentiation, ACTN2 and ACTN3 are upregulated, while ACTN1 and ACTN4 are downregulated. In contrast to sarcomeric isoforms, the non-muscle isoforms have been poorly investigated in developing muscles (39). ACTN4 seems to enhance differentiation of C2C12 myoblasts through the regulation of myogenic genes (38). Here, we show that the loss of Cullin-3 in muscle cells results in accumulation of non-muscle ACTN1. Overexpression of ACTN1 in myoblasts was sufficient to recapitulate most of the phenotype of $s k m$-KO cells, characterized by fusion and AchR clustering defects as well as myofiber hypotrophy, suggesting that ACTN1 and ACTN4 play different, even opposite roles in developing muscles. Contrasting roles for ACTN1 and ACTN4 have already been described in other tissues (65-67), as well as differential biochemical properties in muscles (68). The postsynaptic component of the phenotype of our model reveals roles for Cullin-3-targeted protein turnover and ACTN1 regulation during NMJ formation.

Mutations in Cullin-3 substrate adaptors genes such as KBTBD13 have been associated with early-onset nemaline myopathies. Nemaline myopathies are characterized by nemaline bodies (protein aggregates) in myofibers and are often associated with muscle weakness (69). Proteins constituting nemaline bodies include actin (70), filamin-C (71), telethonin (72), myozenin (73), myopalladin (74), and myotilin (75). While sarcomeric ACTN2 is also thought to be a major constituent $(71,76,77)$, studies investigating whether levels of muscle $\alpha$-actinins are changed in these patients show conflicting results, depending on the method used and whether the employed antibodies have been specific to one $\alpha$-actinin isoform (76-79). To our knowledge, non-muscle ACTN1 and ACTN4 have not been investigated per se in nemaline myopathy patients, but abnormal deposition of ACTN1 proteins in clusters has been observed in cardiomyocytes from patients with dilated cardiomyopathy or chronic pressure overload (80), representing a biomarker for the disease. While these data do not demonstrate that ACTN1 accumulation is causative for the cardiac disease, they are suggestive of an involvement in the pathogenic mechanism. In addition, ACTN1 interacts with UDP- $N$-acetylglucosamine 2-epimerase/ $N$-acetylmannosamine kinase (GNE), an enzyme linked to hereditary inclusion body myopathy (HIBM) (81).

Our data show that ACTN1 and ACTN4 were accumulated in muscle tissues and nemaline bodies of patients with mutations in KBTBD13 but not in TPM2 and Nebulin. The results suggest that accumulation of ACTN1 and ACTN4 may represent a specific pathogenic mechanism due to Cullin-3 impairment.

While further studies are required to determine the extent of non-muscle $\alpha$-actinin expression in nemaline myopathy patients, ACTN1 and ACTN4 should now be considered as constituents of nemaline bodies in some patients.

Indeed, a correlation between ACTN1 or ACTN4 protein levels and severity of the disease would be of clinical interest. An interesting feature of patients with KBTBD13 mutation resides in the lack of a cardiac phenotype (16). In contrast to skeletal muscles, heart development does not rely on cell fusion events. This developmental difference may contribute to protecting the heart from the consequences of ACTN1 accumulation.

To summarize, we propose that Cullin-3 critically contributes to actin cytoskeleton remodeling to allow for optimal myoblast fusion, differentiation, and clustering of AchRs. We propose that non-muscle ACTN1 and ACTN4 are a target for Cullin-3 during myogenesis. Mutations in Cullin-3 substrate adaptors such as KBTBD13 lead to pathological non-muscle actinin accumulation in patients (Figure 11). This finding may represent a new unifying pathogenic mechanism for the etiology of Cullin-3-related myopathies. Further investigations regarding a potential accumulation of non-muscle $\alpha$-actinins in nemaline patients with mutations in other Cullin-3 substrate adaptors (e.g., KLHL9, KLHL40, and $K L H L 41$ ) will be required to confirm this hypothesis. 


\section{Methods}

Generation of skm-KO and iCul3-KO mice. The strategy used to generate the floxed-Cullin-3 mice has been previously described (21). We generated constitutive skeletal muscle-specific (skm-KO) and inducible skeletal muscle-specific (iCul3-KO) Cullin-3 knockouts, by breeding floxed-Cullin-3 mice with mice expressing the Cre recombinase under control of the myogenin promoter (22), or a tetracycline/doxycycline-inducible human skeletal actin (HSA) promoter (82), respectively.

Extraction of total $R N A$, and $R T-P C R$ and $R T-q P C R$ analysis. Mouse muscle samples were snap-frozen in liquid nitrogen and stored at $-80^{\circ} \mathrm{C}$. Cells were washed with PBS, then lysed using TRIzol reagent (Thermo Fisher Scientific). Total RNA from muscles or cells was extracted according to the manufacturer's instructions. Purity of RNA was assessed by a ratio of absorbance at $260 \mathrm{~nm}$ and $230 \mathrm{~nm}>1.7$. 200 ng RNA was used for reverse transcriptase reaction using the Maxima First Strand cDNA Synthesis Kit (Fermentas; Thermo Fisher Scientific) and random hexamers. Oligonucleotides for RT-PCR and RT-qPCR are listed in Supplemental Table 1.

Oligonucleotides optimized for RT-qPCR of murine ACTN1 and cyclophilin B were used in reactions employing the PerfeCTa SYBR Green real-time qPCR mix (Quanta BioSciences) and a CFX96 thermocycler (Bio-Rad). Samples were normalized to cyclophilin B. Unless noted otherwise, 3 biological samples were analyzed per sample group.

C2C12 and primary myoblast cell culture. C2C12 cells were grown and differentiated and their fusion index was assessed as described previously (26).

Mouse primary myoblasts were obtained by dissecting the diaphragm and tongue muscles from E18.5 embryos. Myoblasts were isolated and cultured as previously described (11).

siRNA. Proliferating C2C12 cells were transfected with siRNA directed against Cullin-3 (ON-TARGETplus mouse Cullin-3 [26554]; GE Healthcare Dharmacon), ACTN1 (ON-TARGETplus mouse Actn1 [L-066191-00-0005]), or scrambled control (ON-TARGETplus non-targeting siRNA \#1; GE Healthcare Dharmacon) using DharmaFECT 1 transfection reagent (GE Healthcare Dharmacon) according to the manufacturer's instructions. Twenty-four hours after transfection, cells were changed into differentiation medium and allowed to differentiate for 5 days before analysis. Efficiency of siRNA was verified by immunoblot analysis.

Immunoblot analysis. Proteins were extracted from tissues and cells then quantified as previously described (11). Clarified lysates $(5-10 \mu \mathrm{g})$ were separated by SDS-PAGE. Transfer was performed on nitrocellulose membranes (Bio-Rad). Membranes were incubated with blocking solution (TBS-Tween containing 5\% BSA) for 1 hour at room temperature, then incubated with primary antibodies (Supplemental Table 2) in blocking solution overnight at $4^{\circ} \mathrm{C}$. After washing, secondary HRP-linked antibodies (Dako, Cell Signaling Technology) were applied for 1 hour at room temperature. After washing, antibody-bound proteins were visualized on X-ray films or by Bio-Rad imager. Quantification of band intensities was performed using ImageJ (NIH) software (1.48v) or Bio-Rad Image Lab (v5.2.1). Uncut immunoblot images are shown in the supplemental material.

Quantitative proteome analysis of skeletal muscle. Proteins were isolated from diaphragms by lysis into icecold isolation buffer $(300 \mathrm{mM} \mathrm{KCl}, 30 \mathrm{mM}$ PIPES pH 6.6, 0.5\% NP-40, 1× protease inhibitor [Roche], 1× Phos-Stop [Roche]). Insoluble proteins were removed by centrifugation $\left(20,000 \mathrm{~g}, 10\right.$ minutes at $\left.4^{\circ} \mathrm{C}\right)$, and the supernatant was diluted 1:4 with ice-cold dilution buffer ( $1 \times$ Phos-Stop [Roche], 0.5\% NP-40, $1 \mathrm{mM}$ DTT). Precipitation of actomyosin components was done by centrifugation $\left(20,000 \mathrm{~g}, 15\right.$ minutes at $\left.4^{\circ} \mathrm{C}\right)$, and the remaining supernatant snap-frozen for further analysis by mass spectrometry.

Proteins destined for mass spectrometry were digested by tryptic digest, differentially labeled for iTRAQ, and analyzed in a SciEx QTOF5600 system. Collected spectrograms were further analyzed and quantified using Peaks (83). A full data set of identified proteins was deposited in the Mendeley repository (http://dx. doi.org/10.17632/vwg2bbzw3d.1). Bioinformatic enrichment and pathway analysis was done using Metascape (http://metascape.org/) (84), Morpheus (https://software.broadinstitute.org/morpheus/), BioGRID (https://thebiogrid.org/) (85), and Venny (http://bioinfogp.cnb.csic.es/tools/venny).

Generation of HA-ACTN1 stable cell line. For expression of HA-ACTN1 in C2C12 cells, cDNA was isolated by RT-PCR on mRNA extracted from proliferative $\mathrm{C} 2 \mathrm{C} 12$ cells. Oligonucleotides used for amplification were the following: ACTN1.fwd, GATGGCTAGCATGGACCATTATGATTCCCAG; ACTN1.rev, CATGAGCTCGAGGTCGCTCTCGCCATACAG. PCR products were cloned into a custom-built HA vector (12). HA-ACTN1- or HA control-transfected cells were selected with G-418 (Invitrogen). Plasmid expression was checked by RT-qPCR, immunoblotting, and immunostaining. 
Immunofluorescence microscopy. Cells were rinsed once with PBS, fixed for 15 minutes with $4 \%$ paraformaldehyde, and rinsed 3 times with PBS. Mouse muscles were snap-frozen in isopentane cooled in liquid nitrogen and stored at $-80^{\circ} \mathrm{C}$ until sectioning. Muscles were sectioned using a Leica cryostat. Sections were fixed in ice-cold acetone for 5 minutes, followed by rehydration in $1 \times$ PBS for 10 minutes. Cells or muscle slides were then permeabilized for 10 minutes in $1 \times$ PBS supplemented with $0.2 \%$ Triton X-100 (Sigma Aldrich), washed 3 times with PBS, and incubated in blocking solution (Gold Buffer [GB, $150 \mathrm{mM} \mathrm{NaCl}, 20 \mathrm{mM}$ Tris $\mathrm{pH}$ 7.4] supplemented with $1 \%$ BSA) for 1 hour at room temperature before incubation with primary antibodies (Supplemental Table 2) in blocking solution. Primary antibodies were incubated either for 2 hours at room temperature or overnight at $4^{\circ} \mathrm{C}$. Following incubation, cells were washed 3 times for 5 minutes with PBS and incubated with secondary antibodies (all from Jackson ImmunoResearch Laboratories Inc.) diluted in blocking solution for 1 hour at room temperature. Secondary antibody mixtures also contained DAPI and/or fluorescently linked $\alpha$-bungarotoxin (BGTX; Molecular Probes, Thermo Fisher Scientific), Alexa Fluor 555 conjugate of WGA (Thermo Fisher Scientific), or fluorescently labeled phalloidin (Molecular Probes; Thermo Fisher Scientific) when appropriate. After washing 3 times with PBS for 5 minutes, cells were mounted using fluorescent mounting medium (Dako). Microscopy was performed with an Olympus FV1000 confocal microscope using either the $\times 20$ air objective or the $\times 40$ or $\times 63$ oil immersion objective, and zoom rates between 1 and 3; and imaged in sequential scanning mode. Images were analyzed using ImageJ.

Stimulation of AchR clustering and quantification in C2C12 myotubes. Stimulation and quantification of AchR clustering by rat agrin (R\&D Systems) was performed as described previously (11).

Histological analyses. Transverse sections (10- $\mu \mathrm{m}$ thickness) were stained with H\&E (Sigma-Aldrich) or modified Gomori trichrome (VWR). Diameter and distribution of myofibers were determined. Quantification of fiber numbers, sizes, or nuclear localization was performed on 3 sections separated by at least $30 \mu \mathrm{m}$. Morphometric quantification and evaluation of fiber sizes and numbers were done using ImageJ (1.48v).

Statistics. With the exception for built-in statistics by PEAKS Studio software (Bioinformatics Solutions Inc.), statistical analysis of all data was done by either using ANOVA comparison followed by correction for multiplicity of testing using Bonferroni's multiple-comparisons test, or by unpaired $t$ test, performed using Excel (Microsoft) or GraphPad Prism version 7 for Mac (GraphPad Software). Results are presented as mean \pm SEM. $P$ values less than 0.05 were considered statistically significant. Sample sizes are indicated in the figures or figure legends. If not stated otherwise, both sexes were analyzed in the experimental procedures.

Study approval. Samples from patients and controls were obtained after receiving written informed consent for diagnostic and research investigation. Studies were performed in accordance with the Declaration of Helsinki and approved by the SCHN human ethics committee, 10/CHW/45. All procedures involving genetically modified animals were approved by the UCSD IACUC.

\section{Author contributions}

JB and SL designed research studies. JB, KT, JTS, MG, JML, and MC conducted the experiments and analyzed the data. AB provided human samples. JDS provided animals and reagents. JB and SL wrote the manuscript.

\section{Acknowledgments}

JB is supported by a Muscular Dystrophy Association grant (MDA 515518) and Philippe Foundation grant. Work in the laboratory of SL is supported by an NIH R01 grant (HL128457). MC was supported by the American Heart Association (17UFEL33520004). The work at the mass spectrometry core at UCSD is supported by NIH grants (S10 OD016234, S10 OD021724). We thank the UCSD Microscopy Core and Jennifer Santini, supported by an NIH National Institute of Neurological Disorders and Stroke (NINDS) P30 grant (NS047101). JL was supported by grants from the Association Française contre les Myopathies, Finska Läkaresällskapet, the Medicinska understödsföreningen Liv och Hälsa r.f., and the Magnus Ehrnrooth Foundation. We thank Valeria Marroco, Farah Sheik, Yan Liang, Jing Zhang, Maria Manso, Paul Bushway, and William Bradford, as well as Carina Wallgren-Pettersson and Katarina Pelin for insightful discussions. We also thank Sandra Cooper and Kathryn N. North for providing patient samples and controls for this study, as well as Alan Beggs for providing antibodies.

Address correspondence to: Stephan Lange or Jordan Blondelle, University of California, San Diego, School of Medicine, Division of Cardiology, 9500 Gilman Drive, La Jolla, California 92093-0613C, USA. Phone: 858.822.4618; Email: slange@ucsd.edu (S. Lange); jblondelle@ucsd.edu (J. Blondelle). 
1. Muscaritoli M, Lucia S, Molfino A, Cederholm T, Rossi Fanelli F. Muscle atrophy in aging and chronic diseases: is it sarcopenia or cachexia? Intern Emerg Med. 2013;8(7):553-560.

2. North K. What's new in congenital myopathies? Neuromuscul Disord. 2008;18(6):433-442.

3. Bonaldo P, Sandri M. Cellular and molecular mechanisms of muscle atrophy. Dis Model Mech. 2013;6(1):25-39.

4. Petroski MD, Deshaies RJ. Function and regulation of cullin-RING ubiquitin ligases. Nat Rev Mol Cell Biol. 2005;6(1):9-20.

5. Bosu DR, Kipreos ET. Cullin-RING ubiquitin ligases: global regulation and activation cycles. Cell Div. $2008 ; 3: 7$.

6. Hori T, et al. Covalent modification of all members of human cullin family proteins by NEDD8. Oncogene. 1999;18(48):6829-6834

7. Zhou W, Wei W, Sun Y. Genetically engineered mouse models for functional studies of SKP1-CUL1-F-box-protein (SCF) E3 ubiquitin ligases. Cell Res. 2013;23(5):599-619.

8. Kato JY, Yoneda-Kato N. Mammalian COP9 signalosome. Genes Cells. 2009;14(11):1209-1225.

9. Chen Z, Sui J, Zhang F, Zhang C. Cullin family proteins and tumorigenesis: genetic association and molecular mechanisms J Cancer. 2015;6(3):233-242.

10. Bodine SC, et al. Identification of ubiquitin ligases required for skeletal muscle atrophy. Science. 2001;294(5547):1704-1708.

11. Blondelle J, Shapiro P, Domenighetti AA, Lange S. Cullin E3 ligase activity is required for myoblast differentiation. J Mol Biol. 2017;429(7):1045-1066.

12. Lange S, Perera S, Teh P, Chen J. Obscurin and KCTD6 regulate cullin-dependent small ankyrin-1 (sAnk1.5) protein turnover. Mol Biol Cell. 2012;23(13):2490-2504.

13. Gupta VA, Beggs AH. Kelch proteins: emerging roles in skeletal muscle development and diseases. Skelet Muscle. $2014 ; 4: 11$.

14. Ravenscroft G, et al. Mutations in KLHL40 are a frequent cause of severe autosomal-recessive nemaline myopathy. Am J Hum Genet. 2013;93(1):6-18.

15. Gupta VA, et al. Identification of KLHL41 mutations implicates BTB-Kelch-mediated ubiquitination as an alternate pathway to myofibrillar disruption in nemaline myopathy. Am J Hum Genet. 2013;93(6):1108-1117.

16. Sambuughin N, et al. Dominant mutations in KBTBD13, a member of the BTB/Kelch family, cause nemaline myopathy with cores. Am J Hum Genet. 2010;87(6):842-847.

17. Marttila M, et al. Nebulin interactions with actin and tropomyosin are altered by disease-causing mutations. Skelet Muscle. $2014 ; 4: 15$.

18. Gong W, Gohla RM, Bowlin KM, Koyano-Nakagawa N, Garry DJ, Shi X. Kelch Repeat and BTB domain containing protein 5 (Kbtbd5) regulates skeletal muscle myogenesis through the E2F1-DP1 complex. J Biol Chem. 2015;290(24):15350-15361.

19. Garg A, et al. KLHL40 deficiency destabilizes thin filament proteins and promotes nemaline myopathy. J Clin Invest. 2014;124(8):3529-3539.

20. Papizan JB, Vidal AH, Bezprozvannaya S, Bassel-Duby R, Olson EN. Cullin-3-RING ubiquitin ligase activity is required for striated muscle function in mice. J Biol Chem. 2018;293(23):8802-8811.

21. Singer JD, Gurian-West M, Clurman B, Roberts JM. Cullin-3 targets cyclin E for ubiquitination and controls S phase in mammalian cells. Genes Dev. 1999;13(18):2375-2387.

22. Li S, et al. Requirement for serum response factor for skeletal muscle growth and maturation revealed by tissue-specific gene deletion in mice. Proc Natl Acad Sci USA. 2005;102(4):1082-1087.

23. Liakopoulos D, Doenges G, Matuschewski K, Jentsch S. A novel protein modification pathway related to the ubiquitin system EMBO J. 1998;17(8):2208-2214.

24. Kamitani T, Kito K, Nguyen HP, Yeh ET. Characterization of NEDD8, a developmentally down-regulated ubiquitin-like protein. J Biol Chem. 1997;272(45):28557-28562.

25. Roman W, Gomes ER. Nuclear positioning in skeletal muscle. Semin Cell Dev Biol. 2018;82:51-56.

26. Blondelle J, et al. HACD1, a regulator of membrane composition and fluidity, promotes myoblast fusion and skeletal muscle growth. J Mol Cell Biol. 2015;7(5):429-440.

27. Horsley V, Friday BB, Matteson S, Kegley KM, Gephart J, Pavlath GK. Regulation of the growth of multinucleated muscle cells by an NFATC2-dependent pathway. J Cell Biol. 2001;153(2):329-338.

28. Doherty KR, et al. Normal myoblast fusion requires myoferlin. Development. 2005;132(24):5565-5575.

29. Georgiadis V, et al. Lack of galectin-1 results in defects in myoblast fusion and muscle regeneration. Dev Dyn. 2007;236(4):1014-1024.

30. Laurin M, Fradet N, Blangy A, Hall A, Vuori K, Côté JF. The atypical Rac activator Dock180 (Dock1) regulates myoblast fusion in vivo. Proc Natl Acad Sci USA. 2008;105(40):15446-15451.

31. Hochreiter-Hufford AE, et al. Phosphatidylserine receptor BAI1 and apoptotic cells as new promoters of myoblast fusion. Nature. 2013;497(7448):263-267.

32. Millay DP, et al. Myomaker is a membrane activator of myoblast fusion and muscle formation. Nature. 2013;499(7458):301-305.

33. Lenhart KC, et al. GRAF1 promotes ferlin-dependent myoblast fusion. Dev Biol. 2014;393(2):298-311.

34. Ramirez-Martinez A, et al. KLHL41 stabilizes skeletal muscle sarcomeres by nonproteolytic ubiquitination. Elife. 2017;6:null.

35. Papizan JB, et al. Deficiency in Kelch protein Klhl31 causes congenital myopathy in mice. J Clin Invest. 2017;127(10):3730-3740.

36. Peckham M. Engineering a multi-nucleated myotube, the role of the actin cytoskeleton. J Microsc. 2008;231(3):486-493.

37. Martin SG. Role and organization of the actin cytoskeleton during cell-cell fusion. Semin Cell Dev Biol. 2016;60:121-126.

38. An HT, Kim J, Yoo S, Ko J. Small leucine zipper protein (sLZIP) negatively regulates skeletal muscle differentiation via interaction with $\alpha$-actinin-4. J Biol Chem. 2014;289(8):4969-4979.

39. Sjöblom B, Salmazo A, Djinović-Carugo K. Alpha-actinin structure and regulation. Cell Mol Life Sci. 2008;65(17):2688-2701.

40. Tintignac LA, Brenner HR, Rüegg MA. Mechanisms regulating neuromuscular junction development and function and causes of muscle wasting. Physiol Rev. 2015;95(3):809-852.

41. Natera-de Benito D, et al. KLHL40-related nemaline myopathy with a sustained, positive response to treatment with acetylcholinesterase inhibitors. J Neurol. 2016;263(3):517-523.

42. Dobbins GC, Luo S, Yang Z, Xiong WC, Mei L. alpha-Actinin interacts with rapsyn in agrin-stimulated AChR clustering. Mol 
Brain. 2008;1:18.

43. Dai Z, Luo X, Xie H, Peng HB. The actin-driven movement and formation of acetylcholine receptor clusters. J Cell Biol. 2000;150(6):1321-1334.

44. Li L, et al. Enzymatic activity of the scaffold protein rapsyn for synapse formation. Neuron. 2016;92(5):1007-1019.

45. Sambuughin N, et al. KBTBD13 interacts with Cullin 3 to form a functional ubiquitin ligase. Biochem Biophys Res Commun. 2012;421(4):743-749.

46. DiAntonio A, Hicke L. Ubiquitin-dependent regulation of the synapse. Annu Rev Neurosci. 2004;27:223-246.

47. Feng G, Steinbach JH, Sanes JR. Rapsyn clusters neuronal acetylcholine receptors but is inessential for formation of an interneuronal cholinergic synapse. J Neurosci. 1998;18(11):4166-4176.

48. Nam S, et al. Control of rapsyn stability by the CUL-3-containing E3 ligase complex. J Biol Chem. 2009;284(12):8195-8206

49. Ayyub C. Cullin-5 and cullin-2 play a role in the development of neuromuscular junction and the female germ line of Drosophila. J Genet. 2011;90(2):239-249.

50. Hudson AM, Mannix KM, Cooley L. Actin cytoskeletal organization in drosophila germline ring canals depends on kelch function in a cullin-RING E3 ligase. Genetics. 2015;201(3):1117-1131.

51. Hudson AM, Cooley L. Drosophila Kelch functions with Cullin-3 to organize the ring canal actin cytoskeleton. J Cell Biol. 2010;188(1):29-37.

52. Cartaud A, Stetzkowski-Marden F, Maoui A, Cartaud J. Agrin triggers the clustering of raft-associated acetylcholine receptors through actin cytoskeleton reorganization. Biol Cell. 2011;103(6):287-301.

53. Madhavan R, Peng HB. A synaptic balancing act: local and global signaling in the clustering of ACh receptors at vertebrate neuromuscular junctions. J Neurocytol. 2003;32(5-8):685-696.

54. Vasyutina E, Martarelli B, Brakebusch C, Wende H, Birchmeier C. The small G-proteins Rac1 and Cdc42 are essential for myoblast fusion in the mouse. Proc Natl Acad Sci USA. 2009;106(22):8935-8940.

55. Dalkilic I, Schienda J, Thompson TG, Kunkel LM. Loss of FilaminC (FLNc) results in severe defects in myogenesis and myotube structure. Mol Cell Biol. 2006;26(17):6522-6534.

56. Zhu H, et al. Role of the cofilin 2 gene in regulating the myosin heavy chain genes in mouse myoblast C2C12 cells. Int J Mol Med. 2018;41(2):1096-1102.

57. Keduka E, et al. In vivo characterization of mutant myotilins. Am J Pathol. 2012;180(4):1570-1580.

58. Bloch RJ, Hall ZW. Cytoskeletal components of the vertebrate neuromuscular junction: vinculin, alpha-actinin, and filamin. J Cell Biol. 1983;97(1):217-223.

59. Yorifuji H, Hirokawa N. Cytoskeletal architecture of neuromuscular junction: localization of vinculin. J Electron Microsc Tech. 1989;12(2):160-171.

60. Yasutomi M, Kunishima S, Okazaki S, Tanizawa A, Tsuchida S, Ohshima Y. ACTN1 rod domain mutation associated with congenital macrothrombocytopenia. Ann Hematol. 2016;95(1):141-144.

61. Yao J, et al. Alpha-actinin-4-mediated FSGS: an inherited kidney disease caused by an aggregated and rapidly degraded cytoskeletal protein. PLoS Biol. 2004;2(6):e167.

62. Haywood NJ, et al. Hypertrophic cardiomyopathy mutations in the calponin-homology domain of ACTN2 affect actin binding and cardiomyocyte Z-disc incorporation. Biochem J. 2016;473(16):2485-2493.

63. Ribeiro Ede A, et al. The structure and regulation of human muscle $\alpha$-actinin. Cell. 2014;159(6):1447-1460.

64. Foley KS, Young PW. The non-muscle functions of actinins: an update. Biochem J. 2014;459(1):1-13.

65. Quick Q, Skalli O. Alpha-actinin 1 and alpha-actinin 4: contrasting roles in the survival, motility, and RhoA signaling of astrocytoma cells. Exp Cell Res. 2010;316(7):1137-1147.

66. Shao H, Wu C, Wells A. Phosphorylation of alpha-actinin 4 upon epidermal growth factor exposure regulates its interaction with actin. J Biol Chem. 2010;285(4):2591-2600.

67. Yamaguchi H, et al. Actinin-1 and actinin-4 play essential but distinct roles in invadopodia formation by carcinoma cells. Eur $J$ Cell Biol. 2017;96(7):685-694.

68. Hsu CP, Moghadaszadeh B, Hartwig JH, Beggs AH. Sarcomeric and nonmuscle $\alpha$-actinin isoforms exhibit differential dynamics at skeletal muscle Z-lines. Cytoskeleton (Hoboken). 2018;75(5):213-228.

69. Wallgren-Pettersson C, Sewry CA, Nowak KJ, Laing NG. Nemaline myopathies. Semin Pediatr Neurol. 2011;18(4):230-238.

70. Yamaguchi M, Robson RM, Stromer MH, Dahl DS, Oda T. Nemaline myopathy rod bodies. Structure and composition. J Neurol Sci. 1982;56(1):35-56.

71. Domazetovska A, et al. Intranuclear rod myopathy: molecular pathogenesis and mechanisms of weakness. Ann Neurol. 2007;62(6):597-608

72. Valle G, et al. Telethonin, a novel sarcomeric protein of heart and skeletal muscle. FEBS Lett. 1997;415(2):163-168.

73. Takada F, et al. Myozenin: an alpha-actinin- and gamma-filamin-binding protein of skeletal muscle Z lines. Proc Natl Acad Sci USA. 2001;98(4):1595-1600.

74. Bang ML, et al. Myopalladin, a novel 145-kilodalton sarcomeric protein with multiple roles in Z-disc and I-band protein assemblies. J Cell Biol. 2001;153(2):413-427.

75. Schröder R, et al. Beyond LGMD1A: myotilin is a component of central core lesions and nemaline rods. Neuromuscul Disord. 2003;13(6):451-455

76. Wallgren-Pettersson C, et al. Alpha-actinin in nemaline bodies in congenital nemaline myopathy: immunological confirmation by light and electron microscopy. Neuromuscul Disord. 1995;5(2):93-104.

77. Jockusch BM, Veldman H, Griffiths GW, van Oost BA, Jennekens FG. Immunofluorescence microscopy of a myopathy. alpha-Actinin is a major constituent of nemaline rods. Exp Cell Res. 1980;127(2):409-420.

78. Stuhlfauth I, Jennekens FG, Willemse J, Jockusch BM. Congenital nemaline myopathy. II. Quantitative changes in alpha-actinin and myosin in skeletal muscle. Muscle Nerve. 1983;6(1):69-74.

79. Jockusch BM, Burger MM, DaPrada M, Richards JG, Chaponnier C, Gabbiani G. alpha-Actinin attached to membranes of secretory vesicles. Nature. 1977;270(5638):628-629.

80. Hein S, et al. Deposition of nonsarcomeric alpha-actinin in cardiomyocytes from patients with dilated cardiomyopathy or 
chronic pressure overload. Exp Clin Cardiol. 2009;14(3):e68-e75.

81. Amsili S, et al. UDP-N-acetylglucosamine 2-epimerase/N-acetylmannosamine kinase (GNE) binds to alpha-actinin 1: novel pathways in skeletal muscle? PLoS ONE. 2008;3(6):e2477.

82. Rao P, Monks DA. A tetracycline-inducible and skeletal muscle-specific Cre recombinase transgenic mouse. Dev Neurobiol. 2009;69(6):401-406.

83. Zhang J, et al. PEAKS DB: de novo sequencing assisted database search for sensitive and accurate peptide identification. Mol Cell Proteomics. 2012;11(4):M111.010587.

84. Tripathi S, et al. Meta- and orthogonal integration of influenza "OMICs" data defines a role for UBR4 in virus budding. Cell Host Microbe. 2015;18(6):723-735.

85. Stark C, Breitkreutz BJ, Reguly T, Boucher L, Breitkreutz A, Tyers M. BioGRID: a general repository for interaction datasets. Nucleic Acids Res. 2006;34(Database issue):D535-D539.

86. Schoenauer R, Lange S, Hirschy A, Ehler E, Perriard JC, Agarkova I. Myomesin 3, a novel structural component of the M-band in striated muscle. J Mol Biol. 2008;376(2):338-351. 